\title{
Diiron and diruthenium aminocarbyne complexes containing pseudohalides: stereochemistry and reactivity
}

\author{
Luigi Busetto ${ }^{*}$, Fabio Marchetti, Stefano Zacchini and Valerio Zanotti
}

Dipartimento di Chimica Fisica ed Inorganica, Università di Bologna, Viale Risorgimento 4, I40136 Bologna, Italy

Abstract
Acetonitrile is easily displaced from $\quad\left[\mathrm{Fe}_{2}\{\mu-\mathrm{CN}(\mathrm{Me})(\mathrm{R})\}(\mu-\right.$
$\left.\mathrm{CO})(\mathrm{CO})(\mathrm{MeCN})(\mathrm{Cp})_{2}\right]\left[\mathrm{SO}_{3} \mathrm{CF}_{3}\right]\left(\mathrm{R}=2,6-\mathrm{Me}_{2} \mathrm{C}_{6} \mathrm{H}_{3}(\mathrm{Xyl}), \mathbf{1 a} ; \mathrm{Me}, \mathbf{1 b}\right)$ upon stirring in $\mathrm{THF}$ at room temperature in the presence of $\left[\mathrm{NBu}_{4}\right][\mathrm{SCN}]$. The resulting complexes trans-[ $\mathrm{Fe}_{2}\{\mu-$ $\left.\mathrm{CN}(\mathrm{Me})(\mathrm{R})\}(\mu-\mathrm{CO})(\mathrm{CO})(\mathrm{NCS})(\mathrm{Cp})_{2}\right] \square \quad(\mathrm{R}=\mathrm{Xyl}, \quad$ trans $-\mathbf{2 a} ; \quad \mathrm{Me}$, trans-2b $)$ are completely

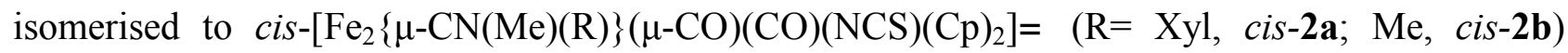
when heated at reflux temperature. Similarly, the complexes cis- $\left[\mathrm{M}_{2}\{\mu-\mathrm{CN}(\mathrm{Me})(\mathrm{R})\}(\mu-\right.$ $\left.\mathrm{CO})(\mathrm{CO})(\mathrm{NCO})(\mathrm{Cp})_{2}\right] \square(\mathrm{M}=\mathrm{Fe}, \mathrm{R}=\mathrm{Me}, \mathbf{4 a} ; \mathrm{M}=\mathrm{Ru}, \mathrm{R}=\mathrm{Xyl}, \mathbf{4 b} ; \mathrm{M}=\mathrm{Ru}, \mathrm{R}=\mathrm{Me}, \mathbf{4 c})$ and cis$\left[\mathrm{M}_{2}\{\mu-\mathrm{CN}(\mathrm{Me})(\mathrm{R})\}(\mu-\mathrm{CO})(\mathrm{CO})\left(\mathrm{N}_{3}\right)(\mathrm{Cp})_{2}\right] \square(\mathrm{M}=\mathrm{Fe}, \mathrm{R}=\mathrm{Xyl}, \mathbf{5 a} ; \mathrm{M}=\mathrm{Fe}, \mathrm{R}=\mathrm{Me}, \mathbf{5 b} ; \mathrm{M}=\mathrm{Ru}, \mathrm{R}$ $=\mathrm{Xyl}, \mathbf{5 c})$ can be obtained by heating at reflux temperature a THF solution of $\left[\mathrm{M}_{2}\{\mu-\right.$ $\left.\mathrm{CN}(\mathrm{Me})(\mathrm{R})\}(\mu-\mathrm{CO})(\mathrm{CO})(\mathrm{MeCN})(\mathrm{Cp})_{2}\right]\left[\mathrm{SO}_{3} \mathrm{CF}_{3}\right](\mathrm{M}=\mathrm{Fe}, \mathrm{R}=\mathrm{Xyl}, \mathbf{1 a} ; \mathrm{M}=\mathrm{Fe}, \mathrm{Me}, \mathbf{1 b} ; \mathrm{M}=\mathrm{Ru}, \mathrm{R}$ $=\mathrm{Xyl}, \mathbf{1} \mathbf{c} ; \mathrm{M}=\mathrm{Ru}, \mathrm{R}=\mathrm{Me}, \mathbf{1 d})$ in the presence of $\mathrm{NaNCO}$ and $\mathrm{NaN}_{3}$, respectively. The reactions of 5 with $\mathrm{MeO}_{2} \mathrm{CC} \equiv \mathrm{CCO}_{2} \mathrm{Me}, \mathrm{HC} \equiv \mathrm{CCO}_{2} \mathrm{Me}$ and $(\mathrm{NC})(\mathrm{H}) \mathrm{C}=\mathrm{C}(\mathrm{H})(\mathrm{CN})$ afford the triazolato complexes $\left[\mathrm{M}_{2}\{\mu-\mathrm{CN}(\mathrm{Me})(\mathrm{R})\}(\mu-\mathrm{CO})(\mathrm{CO})\left\{\mathrm{N}_{3} \mathrm{C}_{2}\left(\mathrm{CO}_{2} \mathrm{Me}\right)_{2}\right\}(\mathrm{Cp})_{2}\right](\mathrm{M}=\mathrm{Fe}, \mathrm{R}=\mathrm{Xyl}, \mathbf{6 a} ; \mathrm{M}=\mathrm{Fe}$, $\mathrm{R}=\mathrm{Me}, \mathbf{6 b} ; \mathrm{M}=\mathrm{Ru}, \mathrm{R}=\mathrm{Xyl}, \mathbf{6 c}),\left[\mathrm{M}_{2}\{\mu-\mathrm{CN}(\mathrm{Me})(\mathrm{R})\}(\mu-\mathrm{CO})(\mathrm{CO})\left\{\mathrm{N}_{3} \mathrm{C}_{2}(\mathrm{H})\left(\mathrm{CO}_{2} \mathrm{Me}\right)\right\}(\mathrm{Cp})_{2}\right](\mathrm{M}$ $=\mathrm{Fe}, \mathrm{R}=\mathrm{Me}, \mathbf{7 a} ; \mathrm{M}=\mathrm{Ru}, \mathrm{R}=\mathrm{Xyl}, \mathbf{7 b})$ and $\left[\mathrm{Fe}_{2}\{\mu-\mathrm{CN}(\mathrm{Me})(\mathrm{Xyl})\}(\mu-\right.$ $\left.\mathrm{CO})(\mathrm{CO})\left\{\mathrm{N}_{3} \mathrm{C}_{2}(\mathrm{H})(\mathrm{CN})\right\}(\mathrm{Cp})_{2}\right], \quad 8$, respectively. The asymmetrically substituted triazolato complexes 7-8 are obtained as mixtures of N(1) and N(2) bonded isomers, whereas 6 exist only in the $\mathrm{N}(2)$ form. Methylation of 6-8 results in the formation of the triazole complexes $\left[\mathrm{Fe}_{2}\{\mu\right.$ $\left.\mathrm{CN}(\mathrm{Me})(\mathrm{Xyl})\}(\mu-\mathrm{CO})(\mathrm{CO})\left\{\mathrm{N}_{3}(\mathrm{Me}) \mathrm{C}_{2}\left(\mathrm{CO}_{2} \mathrm{Me}\right)_{2}\right\}(\mathrm{Cp})_{2}\right]\left[\mathrm{CF}_{3} \mathrm{SO}_{3}\right], \quad$ 9, $\quad\left[\mathrm{M}_{2}\{\mu-\mathrm{CN}(\mathrm{Me})(\mathrm{R})\}(\mu-\right.$ $\left.\mathrm{CO})(\mathrm{CO})\left\{\mathrm{N}_{3}(\mathrm{Me}) \mathrm{C}_{2}(\mathrm{H})\left(\mathrm{CO}_{2} \mathrm{Me}\right)\right\}(\mathrm{Cp})_{2}\right]\left[\mathrm{CF}_{3} \mathrm{SO}_{3}\right](\mathrm{M}=\mathrm{Fe}, \mathrm{R}=\mathrm{Me}, \mathbf{1 0 a} ; \mathrm{M}=\mathrm{Ru}, \mathrm{R}=\mathrm{Xyl}, \mathbf{1 0 b})$ and $\left[\mathrm{Fe}_{2}\{\mu-\mathrm{CN}(\mathrm{Me})(\mathrm{Xyl})\}(\mu-\mathrm{CO})(\mathrm{CO})\left\{\mathrm{N}_{3}(\mathrm{Me}) \mathrm{C}_{2}(\mathrm{H})(\mathrm{CN})\right\}(\mathrm{Cp})_{2}\right]\left[\mathrm{CF}_{3} \mathrm{SO}_{3}\right], \quad$ 11. The crystal structures of trans-2b, $\mathbf{4 b} \cdot \mathbf{C H}_{2} \mathrm{Cl}_{2}, \mathbf{5 a}, \mathbf{6 b} \cdot \mathbf{0 . 5 C} \mathrm{CH}_{2} \mathrm{Cl}_{\mathbf{2}}$ and $\mathbf{8} \cdot \mathbf{C H}_{\mathbf{2}} \mathrm{Cl}_{2}$ have been determined.

\footnotetext{
* Corresponding author. Tel. +39-051-2093694; fax +39-051-2093690.E-mail address: busetto@ms.fci.unibo.it
} 
Keywords: pseudohalides; diiron complexes; diruthenium complexes; aminocarbyne; triazolato complexes.

\section{Introduction}

Pseudohalides are quite versatile ligands, which can coordinate to the metals both terminally or in a bridging fashion $[1,2]$. Moreover, when the two end-atoms are different, they can also display linkage isomerism. If the atoms belong to different periods (e.g NCS', $\mathrm{NCSe}^{-}$), they usually coordinate through nitrogen to 'class a' and via sulfur or selenium to 'class b' metals [3]. Conversely, in the case of isocyanates, $\mathrm{NCO}^{-}$, coordination usually occurs via nitrogen, being $\mathrm{O}$ coordination quite rare $[3 \mathrm{c}, 4,5]$. The coordinated pseudohalides behave as 1,3-dipoles and, therefore, can be used in order to prepare new ligands via 1,3-dipolar cycloaddition reactions [6, 7].

Herein, we report on the synthesis and reactivity of diiron and diruthenium aminocarbyne complexes containing terminal pseudohalides. This work is part of our ongoing interest in the study of the formation of new $\mathrm{C}-\mathrm{C}$ and $\mathrm{C}-\mathrm{N}$ bonds in bimetallic species [8-10], with particular attention to the possibility of selectively addressing the reactions on terminal or bridging ligands and promoting their coupling.

\section{Experimental}

All reactions were carried out routinely under nitrogen using standard Schlenk techniques. Solvents were distilled immediately before use under nitrogen from appropriate drying agents. Infrared spectra were recorded on a Perkin-Elmer Spectrum 2000 FT-IR spectrophotometer and elemental analyses were performed on a ThermoQuest Flash 1112 Series EA Instrument. ESI MS spectra were recorded on a Waters Micromass ZQ 4000 with samples dissolved in $\mathrm{CH}_{3} \mathrm{CN}$. All NMR measurements were performed on Varian Gemini 300 and Mercury Plus 400 instruments. The chemical shifts for ${ }^{1} \mathrm{H}$ and ${ }^{13} \mathrm{C}$ were referenced to internal TMS. The spectra were fully assigned via DEPT experiments and ${ }^{1} \mathrm{H},{ }^{13} \mathrm{C}$ correlation measured using gs-HSQC and gs-HMBC experiments [11]. NOE measurements were recorded using the DPFGSE-NOE sequence [12]. All chemicals were used as received from Aldrich Co., except $\left[\mathrm{Fe}_{2}\{\mu-\mathrm{CN}(\mathrm{Me})(\mathrm{R})\}(\mu-\right.$ $\left.\mathrm{CO})(\mathrm{CO})(\mathrm{MeCN})(\mathrm{Cp})_{2}\right]\left[\mathrm{SO}_{3} \mathrm{CF}_{3}\right]\left(\mathrm{R}=2,6-\mathrm{Me}_{2} \mathrm{C}_{6} \mathrm{H}_{3}(\mathrm{Xyl}), \mathbf{1 a} ; \mathrm{Me}, \mathbf{1 b}\right)$ and $\left[\mathrm{Ru}_{2}\{\mu-\mathrm{CN}(\mathrm{Me})(\mathrm{R})\}(\mu-\right.$ $\left.\mathrm{CO})(\mathrm{CO})(\mathrm{MeCN})(\mathrm{Cp})_{2}\right]\left[\mathrm{SO}_{3} \mathrm{CF}_{3}\right](\mathrm{R}=\mathrm{Xyl}, \mathbf{1 c} ; \mathrm{Me}, 1 \mathbf{1 d})[9 \mathrm{~b}]$ which were prepared by published methods. 
2.1 Synthesis of trans-[Fe $\left.2\{\mu-C N(M e)(R)\}(\mu-C O)(C O)(N C S)(C p)_{2}\right](R=X y l$, trans-2a; Me, trans$2 b)$.

$\left[\mathrm{NBu}_{4}\right][\mathrm{SCN}](129 \mathrm{mg}, 0.430 \mathrm{mmol})$ was added to a $\mathrm{CH}_{2} \mathrm{Cl}_{2}(6 \mathrm{ml})$ solution of $\left[\mathrm{Fe}_{2}\{\mu-\right.$ $\left.\mathrm{CN}(\mathrm{Me})(\mathrm{R})\}(\mu-\mathrm{CO})(\mathrm{CO})(\mathrm{NCMe})(\mathrm{Cp})_{2}\right]\left[\mathrm{CF}_{3} \mathrm{SO}_{3}\right](0.147 \mathrm{mmol})$, and the mixture was stirred at room temperature for 3 hours. The solvent was, then, removed under reduced pressure and the residue was chromatographed through alumina. A fraction containing trans $-\left[\mathrm{Fe}_{2}\{\mu-\mathrm{CN}(\mathrm{Me})(\mathrm{R})\}(\mu-\right.$ $\left.\mathrm{CO})(\mathrm{CO})(\mathrm{NCS})(\mathrm{Cp})_{2}\right] \square$ was obtained using $\mathrm{CH}_{2} \mathrm{Cl}_{2}$ as eluent.

trans-2a Yield $52.4 \mathrm{mg}$ (71 \%). Anal. Calcd. For $\mathrm{C}_{23} \mathrm{H}_{22} \mathrm{Fe}_{2} \mathrm{~N}_{2} \mathrm{O}_{2} \mathrm{~S}: \mathrm{C}, 55.01 ; \mathrm{H}, 4.42 ; \mathrm{N}, 5.58$. Found: C, 55.32; H, 4.12; N, 5.76. IR ( $\left.\mathrm{CH}_{2} \mathrm{Cl}_{2}, 293 \mathrm{~K}\right)$ : v(NCS) 2112 (vs); v(CO) 1968 (vs), 1812 $(\mathrm{s}) ; v(\mu-\mathrm{CN}) 1549(\mathrm{w}) \mathrm{cm}^{-1} .{ }^{1} \mathrm{H}$ NMR $\left(\mathrm{CDCl}_{3}, 293 \mathrm{~K}\right): \delta 7.42-7.29\left(\mathrm{~m}, 3 \mathrm{H}, \mathrm{Me}_{2} \mathrm{C}_{6} \mathrm{H}_{3}\right), 4.93(\mathrm{~s}, 3 \mathrm{H}$, $\mathrm{NMe}), 4.76,4.38(\mathrm{~s}, 10 \mathrm{H}, C p), 2.57,2.41\left(\mathrm{~s}, 6 \mathrm{H}, M_{2} \mathrm{C}_{6} \mathrm{H}_{3}\right) .{ }^{13} \mathrm{C}\left\{{ }^{1} \mathrm{H}\right\} \mathrm{NMR}\left(\mathrm{CDCl}_{3}, 293 \mathrm{~K}\right): \delta 345.5$ $(\mu-C \mathrm{~N}), 262.6(\mu-C \mathrm{O}), 212.5(C \mathrm{O}), 148.7$ (Cipso Xyl), 140.6 (NCS), 134.0, 132.4 (C-Me Xyl), 129.5, 129.3, 128.5 (CH Xyl), 88.9, $87.7(C p), 53.6(\mathrm{NMe}), 18.7,18.4\left(\mathrm{Me}_{2} \mathrm{C}_{6} \mathrm{H}_{3}\right)$.

trans-2b Yield 46.0 mg (76 \%). Anal. Calcd. For $\mathrm{C}_{16} \mathrm{H}_{16} \mathrm{Fe}_{2} \mathrm{~N}_{2} \mathrm{O}_{2} \mathrm{~S}$ : C, 46.63; H, 3.91; N, 6.80. Found: C, 46.87; H, 3.72; N, 6.98. IR ( $\left.\mathrm{CH}_{2} \mathrm{Cl}_{2}, 293 \mathrm{~K}\right)$ : v(NCS) 2115 (vs); v(CO) 1969 (vs), 1809 (s); $v(\mu-\mathrm{CN}) 1568(\mathrm{w}) \mathrm{cm}^{-1} .{ }^{1} \mathrm{H} \mathrm{NMR}\left(\mathrm{CDCl}_{3}, 293 \mathrm{~K}\right): \delta 4.87,4.58(\mathrm{~s}, 10 \mathrm{H}, C p), 4.71,4.31(\mathrm{~s}, 6 \mathrm{H}$, $\mathrm{NMe} 2) .{ }^{13} \mathrm{C}\left\{{ }^{1} \mathrm{H}\right\} \mathrm{NMR}\left(\mathrm{CDCl}_{3}, 293 \mathrm{~K}\right): \delta 340.6(\mu-C \mathrm{~N}), 263.1(\mu-C \mathrm{O}), 211.7(\mathrm{CO}), 140.8(\mathrm{NCS})$, $88.9,87.4(C p), 52.5,51.6\left(\mathrm{NMe}_{2}\right)$.

2.2 Synthesis of cis-[Fe $\left.2\{\mu-C N(M e)(R)\}(\mu-C O)(C O)(N C S)(C p)_{2}\right](R=X y l, c i s-2 a ;$ Me, cis-2b).

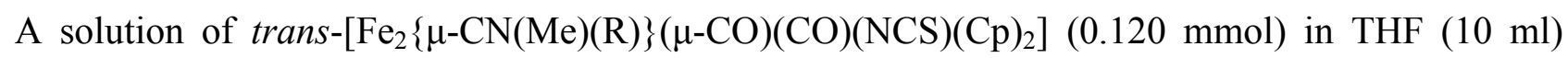
was heated at reflux temperature for 5 hours. The solvent was, then, removed under reduced pressure and the residue was chromatographed through alumina. The final product cis-[Fe $\left\{\mathrm{Fe}_{2}\right.$ $\left.\mathrm{CN}(\mathrm{Me})(\mathrm{R})\}(\mu-\mathrm{CO})(\mathrm{CO})(\mathrm{NCS})(\mathrm{Cp})_{2}\right] \square$ was obtained using THF as eluent.

cis-2a Yield $56.0 \mathrm{mg}\left(93\right.$ \%). Anal. Calcd. For $\mathrm{C}_{23} \mathrm{H}_{22} \mathrm{Fe}_{2} \mathrm{~N}_{2} \mathrm{O}_{2} \mathrm{~S}$ : C, 55.01; H, 4.42; N, 5.58. Found: C, 55.32; H, 4.12; N, 5.76. IR $\left(\mathrm{CH}_{2} \mathrm{Cl}_{2}, 293 \mathrm{~K}\right)$ : v(NCS) 2112 (vs); $v(\mathrm{CO}) 1989$ (vs), 1810 (s); $v(\mu-$ $\mathrm{CN}) 1507(\mathrm{w}) \mathrm{cm}^{-1} .{ }^{1} \mathrm{H}$ NMR $\left(\mathrm{CDCl}_{3}, 293 \mathrm{~K}\right): \delta 7.42-7.25\left(\mathrm{~m}, 3 \mathrm{H}, \mathrm{Me}_{2} \mathrm{C}_{6} H_{3}\right), 4.79,4.17(\mathrm{~s}, 10 \mathrm{H}$, $C p), 4.84$ (s, 3H, NMe), 2.68, 2.27 (s, 6H, Me $\left.\mathrm{C}_{6} \mathrm{H}_{3}\right) .{ }^{13} \mathrm{C}\left\{{ }^{1} \mathrm{H}\right\} \mathrm{NMR}\left(\mathrm{CDCl}_{3}, 293 \mathrm{~K}\right): \delta 341.4(\mu-$ $C \mathrm{~N}), 265.4$ ( $\mu-C \mathrm{O}), 211.5(C \mathrm{O}), 148.1$ (Cipso Xyl), 140.7 (NCS), 135.3-128.2 (C-Me + CH Xyl), 87.3, $86.2(C p), 53.9(\mathrm{~N} M e), 18.5,17.6\left(\mathrm{Me}_{2} \mathrm{C}_{6} \mathrm{H}_{3}\right)$.

cis-2b Yield 39.6 mg (80 \%). Anal. Calcd. For $\mathrm{C}_{16} \mathrm{H}_{16} \mathrm{Fe}_{2} \mathrm{~N}_{2} \mathrm{O}_{2} \mathrm{~S}$ : C, 46.63; H, 3.91; N, 6.80. Found: C, 46.87; H, 3.72; N, 6.98. IR $\left(\mathrm{CH}_{2} \mathrm{Cl}_{2}, 293 \mathrm{~K}\right)$ : $v(\mathrm{NCS}) 2112$ (vs); $v(\mathrm{CO}) 1987$ (vs), 1809 (s); $v(\mu-$ $\mathrm{CN}) 1579(\mathrm{w}) \mathrm{cm}^{-1} .{ }^{1} \mathrm{H} \mathrm{NMR}\left(\mathrm{CDCl}_{3}, 293 \mathrm{~K}\right): \delta 4.74,4.62$ (s, 10H, $\left.C p\right), 4.44,4.13\left(\mathrm{~s}, 6 \mathrm{H}, \mathrm{NMe} e_{2}\right.$. 


\subsection{Synthesis of cis-[Fe $\left.2\{\mu-C N(\mathrm{Me})(\mathrm{Xyl})\}(\mu-\mathrm{CO})(\mathrm{CO})(\mathrm{NCSMe})(\mathrm{Cp})_{2}\right]\left[\mathrm{CF}_{3} \mathrm{SO}_{3}\right]$ (3).}

A solution of $c i s-\left[\mathrm{Fe}_{2}\{\mu-\mathrm{CN}(\mathrm{Me})(\mathrm{Xyl})\}(\mu-\mathrm{CO})(\mathrm{CO})(\mathrm{NCS})(\mathrm{Cp})_{2}\right](131 \mathrm{mg}, 0.260 \mathrm{mmol})$ in $\mathrm{CH}_{2} \mathrm{Cl}_{2}$ $(8 \mathrm{~mL})$ was treated with $\mathrm{CF}_{3} \mathrm{SO}_{3} \mathrm{Me}(0.030 \mathrm{~mL}, 0.26 \mathrm{mmol})$, and the solution stirred for 30 minutes at room temperature. Then, the mixture was filtered on a celite pad, and the solvent was removed under reduced pressure. The residue was washed with diethyl ether $(2 \times 10 \mathrm{~mL})$, yielding a brown powder.

cis-3 Yield $150.0 \mathrm{mg}\left(87 \%\right.$ ). Anal. Calcd. For $\mathrm{C}_{25} \mathrm{H}_{25} \mathrm{~F}_{3} \mathrm{Fe}_{2} \mathrm{~N}_{2} \mathrm{O}_{5} \mathrm{~S}_{2}: \mathrm{C}, 45.07 ; \mathrm{H}, 3.78 ; \mathrm{N}, 4.20$.

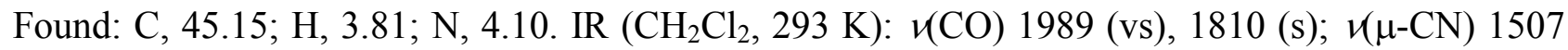
(w) $\mathrm{cm}^{-1} .{ }^{1} \mathrm{H}$ NMR $\left(\mathrm{CDCl}_{3}, 293 \mathrm{~K}\right)$ Isomer $\alpha$ : $\delta 7.42-7.29\left(\mathrm{~m}, 3 \mathrm{H}, \mathrm{Me}_{2} \mathrm{C}_{6} H_{3}\right), 5.04,4.51(\mathrm{~s}, 10 \mathrm{H}$, $C p), 4.79(\mathrm{~s}, 3 \mathrm{H}, \mathrm{NMe}), 2.70,2.13\left(\mathrm{~s}, 6 \mathrm{H}, M_{2} \mathrm{C}_{6} \mathrm{H}_{3}\right), 2.42$ (s, 3H, SMe); Isomer $\beta: \delta 7.42-7.29$ (m, $\left.3 \mathrm{H}, \mathrm{Me}_{2} \mathrm{C}_{6} \mathrm{H}_{3}\right), 5.17,4.34(\mathrm{~s}, 10 \mathrm{H}, C p), 4.50$ (s, 3H, NMe), 2.71, 2.07 (s, 6H, $\left.M e_{2} \mathrm{C}_{6} \mathrm{H}_{3}\right), 2.35$ (s, $3 \mathrm{H}, \mathrm{SMe})$. Isomer ratio $=2 .{ }^{13} \mathrm{C}\left\{{ }^{1} \mathrm{H}\right\} \mathrm{NMR}\left(\mathrm{CDCl}_{3}, 293 \mathrm{~K}\right)$ Isomer $\alpha: \delta 337.2(\mu-C), 263.4(\mu-C \mathrm{O})$, 210.9 (CO), 147.9 (Cipso Xyl), 133.1, 132.0, 129.9, 129.1, 128.9 (C-Me + CH Xyl), 124.1 (NCS), 88.1, $87.7(C p), 54.2(\mathrm{NMe}), 18.7,17.4\left(\mathrm{Me}_{2} \mathrm{C}_{6} \mathrm{H}_{3}\right), 16.7$ (SMe); Isomer $\beta$ : $336.5(\mu-C), 264.1(\mu-$ CO), 210.4 (CO), 148.0 (Cipso Xyl), 133.5, 132.4, 129.8, 129.0 (C-Me + CH Xyl), 123.7 (NCS), 89.1, $86.8(C p), 55.2(\mathrm{NMe}), 18.9,17.8,\left(\mathrm{Me}_{2} \mathrm{C}_{6} \mathrm{H}_{3}\right), 16.6(\mathrm{SMe})$. ESI-MS (ES $\left.{ }^{+}\right): 517\left(\mathrm{M}^{+}, 65 \%\right)$, $444\left(\mathrm{M}^{+}-\mathrm{NCSMe}, 100 \%\right) \mathrm{m} / \mathrm{z}$.

2.4 Synthesis of $\left[M_{2}\{\mu-C N(M e)(R)\}(\mu-C O)(C O)(N C O)(C p)_{2}\right](M=F e, R=M e, 4 a ; M=R u R=X y l$, $4 \boldsymbol{b} ; M=R u, R=M e, 4 c)$.

NaNCO (500 mg, $7.69 \mathrm{mmol})$ was added to a solution of $\left[\mathrm{M}_{2}\{\mathrm{CN}(\mathrm{Me})(\mathrm{R})\}(\mu-\right.$ $\left.\mathrm{CO})(\mathrm{CO})\left(\mathrm{CH}_{3} \mathrm{CN}\right)(\mathrm{Cp})_{2}\right][\mathrm{TfO}](0.230 \mathrm{mmol})$ in THF $(15 \mathrm{ml})$, and the resulting suspension was heated at reflux temperature for 3 hours. Hence, the solvent was removed in vacuo and the residue dissolved in $\mathrm{CH}_{2} \mathrm{Cl}_{2}$ and chromatographed through $\mathrm{Al}_{2} \mathrm{O}_{3}$. A fraction corresponding to the final product $\left[\mathrm{M}_{2}\{\mu-\mathrm{CN}(\mathrm{Me})(\mathrm{R})\}(\mu-\mathrm{CO})(\mathrm{CO})(\mathrm{NCO})(\mathrm{Cp})_{2}\right] \square$ was obtained using $\mathrm{MeCN}$ as eluent.

4a Yield $69.2 \mathrm{mg}$ (76 \%). Anal. Calcd. For $\mathrm{C}_{16} \mathrm{H}_{16} \mathrm{Fe}_{2} \mathrm{~N}_{2} \mathrm{O}_{3}$ : C, 48.52; H, 4.07; N, 7.08. Found: C, 48.23; H, 4.21; N, 6.89. IR $\left(\mathrm{CH}_{2} \mathrm{Cl}_{2}, 293 \mathrm{~K}\right)$ : v(NCO) 2237 (vs); v(CO) 1980 (vs), 1803 (m) cm ${ }^{-1}$. ${ }^{1} \mathrm{H}$ NMR $\left(\mathrm{CDCl}_{3}, 293 \mathrm{~K}\right): \delta 4.77,4.63$ (s, 10H, $\left.C p\right), 4.64,4.23$ (s, 6H, NMe $)_{2}$.

4b Yield 104.7 mg (79 \%). Anal. Calcd. For $\mathrm{C}_{23} \mathrm{H}_{22} \mathrm{~N}_{2} \mathrm{O}_{3} \mathrm{Ru}_{2}$ : C, 47.91; H, 3.85; N, 4.86. Found: C,


$1519(\mathrm{~m}) \mathrm{cm}^{-1} .{ }^{1} \mathrm{H}$ NMR $\left(\mathrm{CDCl}_{3}, 293 \mathrm{~K}\right): \delta 7.29-7.19\left(\mathrm{~m}, 3 \mathrm{H}, \mathrm{Me}_{2} \mathrm{C}_{6} H_{3}\right), 5.16,4.84(\mathrm{~s}, 10 \mathrm{H}, C p)$, $4.25(\mathrm{~s}, 3 \mathrm{H}, \mathrm{N} M e), 2.40,2.29\left(\mathrm{~s}, 6 \mathrm{H}, \mathrm{Me}_{2} \mathrm{C}_{6} \mathrm{H}_{3}\right) .{ }^{13} \mathrm{C}\left\{{ }^{1} \mathrm{H}\right\} \mathrm{NMR}\left(\mathrm{CDCl}_{3}, 293 \mathrm{~K}\right): \delta 313.1(\mu-C \mathrm{~N})$, 235.8 ( $\mu$-CO), 200.1 (CO), 147.9 (Cipso Xyl), 133.7, 132.6 (C-Me Xyl), 129.9, 128.4, 128.1 (CH Xyl), 128.9 (NCO), 88.5, $87.5(C p), 51.5(\mathrm{NMe}), 18.3,17.5\left(\mathrm{Me}_{2} \mathrm{C}_{6} \mathrm{H}_{3}\right)$. 
4c Yield $92.7 \mathrm{mg}(83 \%)$. Anal. Calcd. For $\mathrm{C}_{16} \mathrm{H}_{16} \mathrm{~N}_{2} \mathrm{O}_{3} \mathrm{Ru}_{2}$ : C, 39.51; H, 3.32; N, 5.76. Found: C,

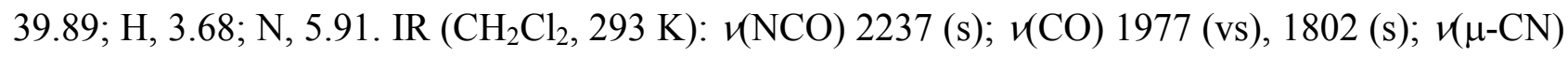
$1589(\mathrm{~m}), 1564(\mathrm{~ms}) \mathrm{cm}^{-1} .{ }^{1} \mathrm{H} \mathrm{NMR}\left(\mathrm{CDCl}_{3}, 293 \mathrm{~K}\right): \delta 5.20,5.02(\mathrm{~s}, 10 \mathrm{H}, C p), 4.02,3.98(\mathrm{~s}, 6 \mathrm{H}$, $\left.\mathrm{N} M e_{2}\right) .{ }^{13} \mathrm{C}\left\{{ }^{1} \mathrm{H}\right\} \mathrm{NMR}\left(\mathrm{CDCl}_{3}, 293 \mathrm{~K}\right): \delta 307.0(\mu-C \mathrm{~N}), 236.2(\mu-C \mathrm{O}), 199.3(C \mathrm{O}), 127.2(\mathrm{NCO})$, 88.5, $87.1(C p), 52.4,51.1\left(\mathrm{NMe}_{2}\right)$.

2.5 Synthesis of $\left[M_{2}\{\mu-C N(M e)(R)\}(\mu-C O)(C O)\left(N_{3}\right)(C p)_{2}\right](M=F e, R=X y l, 5 \boldsymbol{a} ; M=F e R=M e, 5 \boldsymbol{b}$; $M=R u, R=X y l, 5 c)$.

$\mathrm{NaN}_{3} \quad(500 \mathrm{mg}, \quad 7.69 \mathrm{mmol})$ was added to a solution of $\left[\mathrm{M}_{2}\{\mathrm{CN}(\mathrm{Me})(\mathrm{R})\}(\mu-\right.$ $\left.\mathrm{CO})(\mathrm{CO})\left(\mathrm{CH}_{3} \mathrm{CN}\right)(\mathrm{Cp})_{2}\right][\mathrm{TfO}](0.230 \mathrm{mmol})$ in THF $(15 \mathrm{ml})$, and the resulting suspension was heated at reflux temperature for 3 hours. Hence, the solvent was removed in vacuo and the residue dissolved in $\mathrm{CH}_{2} \mathrm{Cl}_{2}$ and chromatographed through $\mathrm{Al}_{2} \mathrm{O}_{3}$. The product $\left[\mathrm{M}_{2}\{\mu-\mathrm{CN}(\mathrm{Me})(\mathrm{R})\}(\mu-\right.$ $\left.\mathrm{CO})(\mathrm{CO})(\mathrm{NCO})(\mathrm{Cp})_{2}\right] \square$ was obtained using THF as eluent.

5a Yield 78.7 mg (70 \%). Anal. Calcd. For $\mathrm{C}_{22} \mathrm{H}_{22} \mathrm{Fe}_{2} \mathrm{~N}_{4} \mathrm{O}_{2}$ : C, 54.36; H, 4.56; N, 11.52. Found: C, 54.57; H, 4.01; N, 11.23. IR $\left(\mathrm{CH}_{2} \mathrm{Cl}_{2}, 293 \mathrm{~K}\right): v\left(\mathrm{~N}_{3}\right) 2033$ (vs); v(CO) 1980 (s), 1800 (br) cm ${ }^{-1} .{ }^{1} \mathrm{H}$ NMR $\left(\mathrm{CDCl}_{3}, 293 \mathrm{~K}\right)$ Isomer $\alpha: \delta 7.40-7.26\left(\mathrm{~m}, 3 \mathrm{H}, \mathrm{Me}_{2} \mathrm{C}_{6} \mathrm{H}_{3}\right), 4.87(\mathrm{~s}, 3 \mathrm{H}, \mathrm{NMe}), 4.80,4.30$ (s, $10 \mathrm{H}, C p), 2.71,2.23\left(\mathrm{~s}, 6 \mathrm{H}, \mathrm{Me}_{2} \mathrm{C}_{6} \mathrm{H}_{3}\right)$; Isomer $\beta: \delta 7.40-7.26\left(\mathrm{~m}, 3 \mathrm{H}, \mathrm{Me}_{2} \mathrm{C}_{6} \mathrm{H}_{3}\right), 5.04(\mathrm{~s}, 3 \mathrm{H}$, $\mathrm{N} M e), 4.85,4.21(\mathrm{~s}, 10 \mathrm{H}, C p), 2.61,2.42\left(\mathrm{~s}, 6 \mathrm{H}, M e_{2} \mathrm{C}_{6} \mathrm{H}_{3}\right) . \alpha / \beta=10 .{ }^{13} \mathrm{C}\left\{{ }^{1} \mathrm{H}\right\} \mathrm{NMR}\left(\mathrm{CDCl}_{3}, 293\right.$ K) Isomer $\alpha$ : $\delta 342.5(\mu-C \mathrm{~N}), 266.1(\mu-C \mathrm{O}), 211.3(C \mathrm{O}), 148.4-128.5(\mathrm{Xyl}), 86.6(C p), 52.3$ $(\mathrm{NMe}), 18.6,17.6\left(\mathrm{Me}_{2} \mathrm{C}_{6} \mathrm{H}_{3}\right)$; Isomer $\beta$ : $86.5(C p), 52.4(\mathrm{NMe})$.

5b Yield 59.2 mg (65 \%). Anal. Calcd. For $\mathrm{C}_{15} \mathrm{H}_{16} \mathrm{Fe}_{2} \mathrm{~N}_{4} \mathrm{O}_{2}$ : C, 45.49; H, 4.07; N, 14.15. Found: C, 45.11; H, 4.23; N, 14.45. IR $\left(\mathrm{CH}_{2} \mathrm{Cl}_{2}, 293 \mathrm{~K}\right): v\left(\mathrm{~N}_{3}\right) 2030(\mathrm{vs}) ; v(\mathrm{CO}) 1976(\mathrm{vs}), 1806(\mathrm{~s}) ; v(\mu-\mathrm{CN})$ $1578(\mathrm{~m}) \mathrm{cm}^{-1} .{ }^{1} \mathrm{H}$ NMR $\left(\mathrm{CDCl}_{3}, 293 \mathrm{~K}\right): \delta 4.74,4.63$ (s, 10H, Cp), 4.66, 4.24 (s, 6H, NMe $)_{2}$. ${ }^{13} \mathrm{C}\left\{{ }^{1} \mathrm{H}\right\} \mathrm{NMR}\left(\mathrm{CDCl}_{3}, 293 \mathrm{~K}\right): \delta 89.1,86.6(C p), 52.8,51.6\left(\mathrm{NMe}_{2}\right)$.

5c Yield 99.5 mg (75 \%). Anal. Calcd. For $\mathrm{C}_{22} \mathrm{H}_{22} \mathrm{~N}_{4} \mathrm{O}_{2} \mathrm{Ru}_{2}$ : C, 45.83; H, 3.85; N, 9.72. Found: C, 46.15; H, 3.61; N, 9.91. IR $\left(\mathrm{CH}_{2} \mathrm{Cl}_{2}, 293 \mathrm{~K}\right)$ : $v\left(\mathrm{~N}_{3}\right) 2033$ (vs); $v(\mathrm{CO}) 1974$ (s), 1800 (s); $v(\mu-\mathrm{CN})$ $1512(\mathrm{~m}) \mathrm{cm}^{-1} .{ }^{1} \mathrm{H}$ NMR $\left(\mathrm{CDCl}_{3}, 293 \mathrm{~K}\right): \delta 7.29-7.20\left(\mathrm{~m}, 3 \mathrm{H}, \mathrm{Me}_{2} \mathrm{C}_{6} H_{3}\right), 5.21,4.83(\mathrm{~s}, 10 \mathrm{H}, C p)$, 4.30 (s, 3H, NMe), 2.39, $2.32\left(\mathrm{~s}, 6 \mathrm{H}, M_{2} \mathrm{C}_{6} \mathrm{H}_{3}\right) .{ }^{13} \mathrm{C}\left\{{ }^{1} \mathrm{H}\right\} \mathrm{NMR}\left(\mathrm{CDCl}_{3}, 293 \mathrm{~K}\right): \delta 312.1(\mu-C \mathrm{~N})$, 237.0 ( $\mu$-CO), 200.3 (CO), 147.9 (Cipso Xyl), 133.7, 132.6 (C-Me Xyl), 129.7, 128.3, $128.0(C \mathrm{H}$ $\mathrm{Xyl}), 88.5,87.8(C p), 51.3(\mathrm{NMe}), 18.2,17.5\left(\mathrm{Me}_{2} \mathrm{C}_{6} \mathrm{H}_{3}\right)$.

2.6 Synthesis of $\left[M_{2}\{\mu-C N(M e)(R)\}(\mu-C O)(C O)\left\{N_{3} C_{2}\left(C_{2} \mathrm{Me}_{2}\right\}(C p)_{2}\right](M=F e, R=X y l, \boldsymbol{6 a} ; M=\right.$ $F e R=M e, \boldsymbol{6} \boldsymbol{b} ; M=R u, R=X y l, \boldsymbol{6} \boldsymbol{c})$. 
A solution containing $\left[\mathrm{M}_{2}\{\mu-\mathrm{CN}(\mathrm{Me})(\mathrm{R})\}(\mu-\mathrm{CO})(\mathrm{CO})\left(\mathrm{N}_{3}\right)(\mathrm{Cp})_{2}\right] \quad(0.411 \mathrm{mmol})$ and $\mathrm{MeO}_{2} \mathrm{CC} \equiv \mathrm{CCO}_{2} \mathrm{Me}(0.2 \mathrm{ml}, 1.63 \mathrm{mmol})$ in THF $(15 \mathrm{ml})$ was heated at reflux temperature for 3 hours and, then, the solvent was removed under reduced pressure. Hence, the residue was washed with petroleum ether $(2 \times 20 \mathrm{ml})$, dissolved in $\mathrm{CH}_{2} \mathrm{Cl}_{2}$ and chromatographed through $\mathrm{Al}_{2} \mathrm{O}_{3}$. The final product was obtained as an orange-brown fraction using THF as eluent.

6a Yield 206.6 mg (70 \%). Anal. Calcd. For $\mathrm{C}_{28} \mathrm{H}_{28} \mathrm{Fe}_{2} \mathrm{~N}_{4} \mathrm{O}_{6}$ : C, 53.53; H, 4.49; N, 8.92. Found: C, 53.89; H, 4.06; N, 8.68. IR $\left(\mathrm{CH}_{2} \mathrm{Cl}_{2}, 293 \mathrm{~K}\right): v(\mathrm{CO}) 1985$ (vs), 1806 (s); $v\left(\mathrm{CO}_{2} \mathrm{Me}\right) 1734$ (s); $v(\mu-$ $\mathrm{CN}) 1507(\mathrm{~m}) \mathrm{cm}^{-1} .{ }^{1} \mathrm{H}$ NMR $\left(\mathrm{CDCl}_{3}, 293 \mathrm{~K}\right): \delta 7.31-7.23\left(\mathrm{~m}, 3 \mathrm{H}, \mathrm{Me}_{2} \mathrm{C}_{6} \mathrm{H}_{3}\right), 4.96(\mathrm{~s}, 3 \mathrm{H}, \mathrm{NMe})$, 4.85, 4.28 (s, 10H, Cp), $3.72\left(\mathrm{~s}, 6 \mathrm{H}, \mathrm{CO}_{2} \mathrm{Me}\right), 2.68,2.15\left(\mathrm{~s}, 6 \mathrm{H}, M_{2} \mathrm{C}_{6} \mathrm{H}_{3}\right) .{ }^{13} \mathrm{C}\left\{{ }^{1} \mathrm{H}\right\} \mathrm{NMR}\left(\mathrm{CDCl}_{3}\right.$, $293 \mathrm{~K}): \delta 340.4(\mu-C \mathrm{~N}), 265.9(\mu-C \mathrm{O}), 210.6(C \mathrm{O}), 162.1\left(\mathrm{CO}_{2} \mathrm{Me}\right), 148.9$ (Cipso Xyl), 140.5 $\left(\mathrm{N}_{3} C_{2}\right), 134.8,132.6$ (C-Me Xyl), 130.2, 128.3, 128.1 (CH Xyl), 87.3, $86.9(C p), 53.2(\mathrm{~N} M e), 51.5$ $\left(\mathrm{CO}_{2} \mathrm{Me}\right), 18.6,17.1\left(\mathrm{Me}_{2} \mathrm{C}_{6} \mathrm{H}_{3}\right)$.

6b Yield $168.1 \mathrm{mg}\left(76 \%\right.$ ). Anal. Calcd. For $\mathrm{C}_{21} \mathrm{H}_{22} \mathrm{Fe}_{2} \mathrm{~N}_{4} \mathrm{O}_{6}$ : C, 46.87; H, 4.12; N, 10.41. Found: C, 47.05; H, 3.98; N, 10.07. IR $\left(\mathrm{CH}_{2} \mathrm{Cl}_{2}, 293 \mathrm{~K}\right): v(\mathrm{CO}) 1983$ (vs), 1806 (s); $v\left(\mathrm{CO}_{2} \mathrm{Me}\right) 1727(\mathrm{~s}) ; v(\mu-$ CN) $1577(\mathrm{w}), 1551(\mathrm{~m}) \mathrm{cm}^{-1} .{ }^{1} \mathrm{H} \mathrm{NMR}\left(\mathrm{CDCl}_{3}, 293 \mathrm{~K}\right): \delta 4.73,4.71(\mathrm{~s}, 10 \mathrm{H}, C p), 4.68,4.11(\mathrm{~s}$, $\left.6 \mathrm{H}, \mathrm{NMe} e_{2}\right), 3.69\left(\mathrm{~s}, 6 \mathrm{H}, \mathrm{CO}_{2} \mathrm{Me}\right) .{ }^{13} \mathrm{C}\left\{{ }^{1} \mathrm{H}\right\} \mathrm{NMR}\left(\mathrm{CDCl}_{3}, 293 \mathrm{~K}\right): \delta 334.3(\mu-\mathrm{CN}), 265.9(\mu-C \mathrm{O})$, $210.0(\mathrm{CO}), 162.1\left(\mathrm{CO}_{2} \mathrm{Me}\right), 140.2\left(\mathrm{~N}_{3} \mathrm{C}_{2}\right), 87.0,86.9(\mathrm{Cp})$, 53.3, $52.2(\mathrm{NMe}), 51.6\left(\mathrm{CO}_{2} \mathrm{Me}\right)$.

6c Yield 254.4 mg (86 \%). Anal. Calcd. For $\mathrm{C}_{28} \mathrm{H}_{28} \mathrm{~N}_{4} \mathrm{O}_{6} \mathrm{Ru}_{2}$ : C, 46.79; H, 3.93; N, 7.80. Found: C, 46.24; H, 4.11; N, 7.99. IR ( $\left.\mathrm{CH}_{2} \mathrm{Cl}_{2}, 293 \mathrm{~K}\right)$ : v(CO) 1979 (vs), 1804 (s); v( $\left.\mathrm{CO}_{2} \mathrm{Me}\right) 1738$ (sh), 1713 (vs); $v(\mu-\mathrm{CN}) 1519(\mathrm{~m}) \mathrm{cm}^{-1} .{ }^{1} \mathrm{H}$ NMR $\left(\mathrm{CDCl}_{3}, 293 \mathrm{~K}\right): \delta 7.31-7.20\left(\mathrm{~m}, 3 \mathrm{H}, \mathrm{Me}_{2} \mathrm{C}_{6} \mathrm{H}_{3}\right), 5.33,4.85$ (s, 10H, Cp), 4.44 (s, 3H, NMe), 3.83 (s, 6H, $\left.\mathrm{CO}_{2} M e\right), 2.44,2.22\left(\mathrm{~s}, 6 \mathrm{H}, M e_{2} \mathrm{C}_{6} \mathrm{H}_{3}\right) .{ }^{13} \mathrm{C}\left\{{ }^{1} \mathrm{H}\right\} \mathrm{NMR}$ $\left(\mathrm{CDCl}_{3}, 293 \mathrm{~K}\right): \delta 311.4(\mu-C \mathrm{~N}), 236.3(\mu-C \mathrm{O}), 199.0(\mathrm{CO}), 162.2\left(\mathrm{CO}_{2} \mathrm{Me}\right), 148.3$ (Cipso Xyl), $139.7\left(\mathrm{~N}_{3} C_{2}\right), 134.3,132.3$ (C-Me Xyl), 129.9, 128.2, 127.7 (CH Xyl), 88.6, 88.1 (Cp), $52.1(\mathrm{~N} M e)$, $51.6\left(\mathrm{CO}_{2} \mathrm{Me}\right), 18.4,17.1\left(\mathrm{Me}_{2} \mathrm{C}_{6} \mathrm{H}_{3}\right)$

2.7 Synthesis of $\left[\mathrm{M}_{2}\{\mu-\mathrm{CN}(\mathrm{Me})(\mathrm{R})\}(\mu-\mathrm{CO})(\mathrm{CO})\left\{\mathrm{N}_{3} \mathrm{C}_{2}(\mathrm{H})\left(\mathrm{CO}_{2} \mathrm{Me}\right)\right\}(\mathrm{Cp})_{2}\right](\mathrm{M}=\mathrm{Fe}, \mathrm{R}=\mathrm{Me}, 7 \boldsymbol{a} ; \mathrm{M}=$ $R u, R=X y l, 7 \boldsymbol{b})$.

A solution containing $\left[\mathrm{M}_{2}\{\mu-\mathrm{CN}(\mathrm{Me})(\mathrm{R})\}(\mu-\mathrm{CO})(\mathrm{CO})\left(\mathrm{N}_{3}\right)(\mathrm{Cp})_{2}\right](0.240 \mathrm{mmol})$ and $\mathrm{HC} \equiv \mathrm{CCO}_{2} \mathrm{Me}$ $(0.1 \mathrm{ml}, 1.12 \mathrm{mmol})$ in THF $(15 \mathrm{ml})$ was heated at reflux temperature for 3 hours and, then, the solvent was removed under reduced pressure. Hence, the residue was washed with petroleum ether $(2 \times 20 \mathrm{ml})$, dissolved in $\mathrm{CH}_{2} \mathrm{Cl}_{2}$ and chromatographed through $\mathrm{Al}_{2} \mathrm{O}_{3}$. The final product was obtained as an orange-brown fraction using THF as eluent.

7a Yield 93.5 mg (81 \%). Anal. Calcd. For $\mathrm{C}_{19} \mathrm{H}_{20} \mathrm{Fe}_{2} \mathrm{~N}_{4} \mathrm{O}_{4}$ : C, 47.53; H, 4.20; $\mathrm{N}, 11.67$. Found: C, 47.86; H, 4.01; N, 11.12. IR $\left(\mathrm{CH}_{2} \mathrm{Cl}_{2}, 293 \mathrm{~K}\right): v(\mathrm{CO}) 1982(\mathrm{vs}), 1805(\mathrm{~s}) ; \gamma\left(\mathrm{CO}_{2} \mathrm{Me}\right) 1721(\mathrm{~s}) ; \gamma(\mu-$ 
CN) $1576(\mathrm{~ms}), 1505(\mathrm{w}) \mathrm{cm}^{-1} .{ }^{1} \mathrm{H}$ NMR $\left(\mathrm{CDCl}_{3}, 293 \mathrm{~K}\right)$ Major isomer: $\delta 7.56(\mathrm{~s}, 1 \mathrm{H}, \mathrm{CH}), 4.82$, $4.78(\mathrm{~s}, 10 \mathrm{H}, C p), 4.70,4.21\left(\mathrm{~s}, 6 \mathrm{H}, \mathrm{N} M e_{2}\right), 3.73\left(\mathrm{~s}, 3 \mathrm{H}, \mathrm{CO}_{2} \mathrm{Me}\right)$; Minor isomer: $\delta 7.72(\mathrm{~s}, 1 \mathrm{H}$, $\mathrm{CH})$, 4.91, $4.81(\mathrm{~s}, 10 \mathrm{H}, \mathrm{Cp}), 4.70,4.21\left(\mathrm{~s}, 6 \mathrm{H}, \mathrm{NMe}\right.$ ), $3.76\left(\mathrm{~s}, 3 \mathrm{H}, \mathrm{CO}_{2} \mathrm{Me}\right)$. Major/Minor $=2$. ${ }^{13} \mathrm{C}\left\{{ }^{1} \mathrm{H}\right\}$ NMR $\left(\mathrm{CDCl}_{3}, 293 \mathrm{~K}\right)$ Major isomer: $\delta 334.3(\mu-C \mathrm{~N}), 266.6(\mu-C \mathrm{O}), 210.3(C \mathrm{O}), 162.4$ $\left(\mathrm{CO}_{2} \mathrm{Me}\right), 138.2\left(\mathrm{~N}_{3} \mathrm{CHCCO}_{2} \mathrm{Me}\right), 137.6(\mathrm{CH}), 87.0,86.9(\mathrm{Cp}), 53.2,52.2\left(\mathrm{NMe}_{2}\right), 50.8\left(\mathrm{CO}_{2} \mathrm{Me}\right)$; Minor isomer: $\delta 332.5(\mu-C \mathrm{~N}), 270.7(\mu-C \mathrm{O}), 209.6(C \mathrm{O}), 162.2\left(\mathrm{CO}_{2} \mathrm{Me}\right), 139.7\left(\mathrm{~N}_{3} \mathrm{CHCCO}_{2} \mathrm{Me}\right)$, 139.4 (CH), 87.1, $86.7(C p), 53.5,52.8\left(\mathrm{NMe}_{2}\right), 50.9\left(\mathrm{CO}_{2} \mathrm{Me}\right)$.

7b Yield 128.7 mg (81 \%). Anal. Calcd. For $\mathrm{C}_{26} \mathrm{H}_{26} \mathrm{~N}_{4} \mathrm{O}_{4} \mathrm{Ru}_{2}$ : C, 47.27; H, 3.97; N, 8.48. Found: C, 47.65; H, 3.68; N, 8.89. IR $\left(\mathrm{CH}_{2} \mathrm{Cl}_{2}, 293 \mathrm{~K}\right)$ : $v(\mathrm{CO}) 1978$ (s), 1803 (s); $v\left(\mathrm{CO}_{2} \mathrm{Me}\right) 1712(\mathrm{~s}) ; \gamma(\mu-$ $\mathrm{CN}) 1520(\mathrm{~ms}), 1505(\mathrm{w}) \mathrm{cm}^{-1} .{ }^{1} \mathrm{H} \mathrm{NMR}\left(\mathrm{CDCl}_{3}, 293 \mathrm{~K}\right)$ Major isomer: $\delta 7.86(\mathrm{~s}, 1 \mathrm{H}, \mathrm{CH}), 7.27-$ $7.22\left(\mathrm{~m}, 3 \mathrm{H}, \mathrm{Me}_{2} \mathrm{C}_{6} \mathrm{H}_{3}\right), 5.25,4.86(\mathrm{~s}, 10 \mathrm{H}, \mathrm{Cp}), 4.52$ (s, 3H, NMe), 3.83 (s, 3H, $\left.\mathrm{CO}_{2} \mathrm{Me}\right), 2.46,2.18$ $\left(\mathrm{s}, 6 \mathrm{H}, \mathrm{Me}_{2} \mathrm{C}_{6} \mathrm{H}_{3}\right)$; Minor isomer: $\delta 7.79(\mathrm{~s}, 1 \mathrm{H}, \mathrm{CH}), 7.27-7.22\left(\mathrm{~m}, 3 \mathrm{H}, \mathrm{Me}_{2} \mathrm{C}_{6} \mathrm{H}_{3}\right), 5.33,4.86(\mathrm{~s}$, 10H, Cp), 4.48 (s, 3H, NMe), 3.79 (s, 3H, $\left.\mathrm{CO}_{2} M e\right), 2.46,2.19$ (s, 6H, $\mathrm{Me}_{2} \mathrm{C}_{6} \mathrm{H}_{3}$ ). Major/Minor $=2$. ${ }^{13} \mathrm{C}\left\{{ }^{1} \mathrm{H}\right\}$ NMR $\left(\mathrm{CDCl}_{3}, 293 \mathrm{~K}\right)$ Major isomer: $\delta 310.7(\mu-C \mathrm{~N}), 240.1(\mu-C \mathrm{O}), 199.1(C \mathrm{O}), 162.9$ $\left(C \mathrm{O}_{2} \mathrm{Me}\right), 148.5$ (Cipso Xyl), $139.5(\mathrm{CH}), 137.3\left(\mathrm{~N}_{3} \mathrm{CHCCO}_{2} \mathrm{Me}\right), 134.5,132.3(C-\mathrm{Me} \mathrm{Xyl}), 130.0$ 127.8 (CH Xyl), 88.7, $88.0(C p), 52.5(\mathrm{NMe}), 51.0\left(\mathrm{CO}_{2} \mathrm{Me}\right), 18.3,17.3\left(\mathrm{Me}_{2} \mathrm{C}_{6} \mathrm{H}_{3}\right)$; Minor isomer: $\delta 311.9(\mu-C \mathrm{~N}), 237.3(\mu-C \mathrm{O}), 199.5(C \mathrm{O}), 162.8\left(\mathrm{CO}_{2} \mathrm{Me}\right), 148.5$ (Cipso Xyl), $139.4(C \mathrm{H}), 138.1$ $\left(\mathrm{N}_{3} \mathrm{CHCCO}_{2} \mathrm{Me}\right), 134.5,132.5$ (C-Me Xyl), 130.0-127.8 (CH Xyl), 88.6, 88.2 (Cp), 52.0 (NMe), $51.0\left(\mathrm{CO}_{2} \mathrm{Me}\right), 18.3,16.9\left(\mathrm{Me}_{2} \mathrm{C}_{6} \mathrm{H}_{3}\right)$.

\subsection{Synthesis of $\left[\mathrm{Fe}_{2}\{\mu-\mathrm{CN}(\mathrm{Me})(\mathrm{Xyl})\}(\mu-\mathrm{CO})(\mathrm{CO})\left\{\mathrm{N}_{3} \mathrm{C}_{2}(\mathrm{H})(\mathrm{CN})\right\}(\mathrm{Cp})_{2}\right], \boldsymbol{8}$.}

A solution containing $\left[\mathrm{Fe}_{2}\{\mu-\mathrm{CN}(\mathrm{Me})(\mathrm{Xyl})\}(\mu-\mathrm{CO})(\mathrm{CO})\left(\mathrm{N}_{3}\right)(\mathrm{Cp})_{2}\right](75.1 \mathrm{mg}, 0.154 \mathrm{mmol})$ and $(\mathrm{NC})(\mathrm{H}) \mathrm{C} \equiv \mathrm{C}(\mathrm{H})(\mathrm{CN})(34.1 \mathrm{mg}, 0.437 \mathrm{mmol})$ in THF $(6 \mathrm{ml})$ was heated at reflux temperature for 5 hours and, then, the solvent was removed under reduced pressure. Hence, the residue was washed with petroleum ether $(2 \times 20 \mathrm{ml})$, dissolved in $\mathrm{CH}_{2} \mathrm{Cl}_{2}$ and chromatographed through $\mathrm{Al}_{2} \mathrm{O}_{3}$. The final product was obtained as an orange-brown fraction using THF as eluent.

8 Yield $67.8 \mathrm{mg}(82 \%)$. Anal. Calcd. For $\mathrm{C}_{25} \mathrm{H}_{23} \mathrm{Fe}_{2} \mathrm{~N}_{5} \mathrm{O}_{2}$ : C, 55.90; H, 4.32; N, 13.04. Found: C, 55.59; H, 4.54; N, 13.15. IR $\left(\mathrm{CH}_{2} \mathrm{Cl}_{2}, 293 \mathrm{~K}\right)$ : $v(\mathrm{CN}) 2232(\mathrm{~ms}) ; v(\mathrm{CO}) 1986(\mathrm{vs}), 1805$ (s); $v(\mu-$ $\mathrm{CN}) 1509(\mathrm{~ms}), 1505(\mathrm{w}) \mathrm{cm}^{-1} .{ }^{1} \mathrm{H}$ NMR $\left(\mathrm{CDCl}_{3}, 293 \mathrm{~K}\right)$ Major isomer: $\delta 7.74(\mathrm{~s}, 1 \mathrm{H}, \mathrm{CH}), 7.31-$ $7.21\left(\mathrm{~m}, 3 \mathrm{H}, \mathrm{Me}_{2} \mathrm{C}_{6} H_{3}\right), 5.11(\mathrm{~s}, 3 \mathrm{H}, \mathrm{NMe}), 4.74,4.29$ (s, 10H, Cp), 2.69, $2.03\left(\mathrm{~s}, 6 \mathrm{H}, \mathrm{Me}_{2} \mathrm{C}_{6} \mathrm{H}_{3}\right)$; Minor isomer: $\delta 7.46(\mathrm{~s}, 1 \mathrm{H}, \mathrm{CH}), 7.31-7.21\left(\mathrm{~m}, 3 \mathrm{H}, \mathrm{Me}_{2} \mathrm{C}_{6} \mathrm{H}_{3}\right), 4.96(\mathrm{~s}, 3 \mathrm{H}, \mathrm{NMe}), 4.81,4.28(\mathrm{~s}$, 10H, Cp), 2.68, $2.08\left(\mathrm{~s}, 6 \mathrm{H}, \mathrm{Me}_{2} \mathrm{C}_{6} \mathrm{H}_{3}\right)$. Major/Minor $=1.1 .{ }^{13} \mathrm{C}\left\{{ }^{1} \mathrm{H}\right\} \mathrm{NMR}\left(\mathrm{CDCl}_{3}, 293 \mathrm{~K}\right)$ Major isomer: $\delta 339.2(\mu-C \mathrm{~N}), 270.9(\mu-C \mathrm{O}), 210.1(C \mathrm{O}), 148.9$ (Cipso Xyl), $140.9(C \mathrm{H}), 134.5,132.4$ (C-Me Xyl), 130.4-128.2 (CH Xyl), $118.5(C \mathrm{~N}), 114.4\left(\mathrm{~N}_{3} \mathrm{CHCCN}\right), 87.2,86.9(C p), 53.9(\mathrm{NMe})$, 
18.6, $17.2\left(\mathrm{Me}_{2} \mathrm{C}_{6} \mathrm{H}_{3}\right)$; Minor isomer: $\delta 340.9(\mu-\mathrm{CN}), 266.7(\mu-\mathrm{CO}), 210.8(\mathrm{CO}), 149.0$ (Cipso $\mathrm{Xyl}), 139.2(\mathrm{CH}), 134.5,132.6$ (C-Me Xyl), 130.4-128.2 (CH Xyl), $119.4(C \mathrm{~N}), 114.5$ $\left(\mathrm{N}_{3} \mathrm{CHCCN}\right), 87.3,86.0(C p), 53.1(\mathrm{NMe}), 18.6,16.9\left(\mathrm{Me}_{2} \mathrm{C}_{6} \mathrm{H}_{3}\right)$.

\subsection{Synthesis of $\left[\mathrm{Fe}_{2}\{\mu-\mathrm{CN}(\mathrm{Me})(\mathrm{Xyl})\}(\mu-\mathrm{CO})(\mathrm{CO})\left\{\mathrm{N}_{3}(\mathrm{Me}) \mathrm{C}_{2}\left(\mathrm{CO}_{2} \mathrm{Me}\right)_{2}\right\}(\mathrm{Cp})_{2}\right]\left[\mathrm{CF}_{3} \mathrm{SO}_{3}\right], \mathbf{9}$.}

$\mathrm{CF}_{3} \mathrm{SO}_{3} \mathrm{Me}(0.1 \mathrm{ml}, 0.884 \mathrm{mmol})$ was added to a solution of $\mathbf{6 a}(200.0 \mathrm{mg}, 0.279 \mathrm{mmol})$ in $\mathrm{CH}_{2} \mathrm{Cl}_{2}$ (6 $\mathrm{ml})$; after stirring at room temperature for 30 minutes, the solvent was removed under reduced pressure and the residue washed with $\mathrm{Et}_{2} \mathrm{O}(2 \times 10 \mathrm{ml})$.

9 Yield 222.6 mg (92 \%). Anal. Calcd. For $\mathrm{C}_{30} \mathrm{H}_{31} \mathrm{~F}_{3} \mathrm{Fe}_{2} \mathrm{~N}_{4} \mathrm{O}_{9} \mathrm{~S}: \mathrm{C}, 41.55 ; \mathrm{H}, 3.60 ; \mathrm{N}, 6.46$. Found: C, 41.92; H, 3.47; N, 6.31. IR $\left(\mathrm{CH}_{2} \mathrm{Cl}_{2}, 293 \mathrm{~K}\right)$ : $v(\mathrm{CO}) 1982(\mathrm{vs}), 1808(\mathrm{~s}) ; \gamma\left(\mathrm{CO}_{2} \mathrm{Me}\right) 1745(\mathrm{~s}) \mathrm{cm}^{-}$ 1. ${ }^{1} \mathrm{H}$ NMR $\left(\mathrm{CDCl}_{3}, 293 \mathrm{~K}\right): \delta 7.25\left(\mathrm{~m}, 3 \mathrm{H}, \mathrm{Me}_{2} \mathrm{C}_{6} H_{3}\right), 5.06$ (s, 3H, NMe), 4.90, $4.28(\mathrm{~s}, 10 \mathrm{H}, C p)$, $3.98\left(\mathrm{~s}, 3 \mathrm{H}, \mathrm{N}_{3} \mathrm{Me}\right), 3.80\left(\mathrm{~s}, 6 \mathrm{H}, \mathrm{CO}_{2} \mathrm{Me}\right), 2.62,2.15\left(\mathrm{~s}, 6 \mathrm{H}, M_{2} \mathrm{C}_{6} \mathrm{H}_{3}\right) .{ }^{13} \mathrm{C}\left\{{ }^{1} \mathrm{H}\right\} \mathrm{NMR}\left(\mathrm{CDCl}_{3}, 293\right.$ $\mathrm{K}): \delta 330.4(\mu-C \mathrm{~N}), 266.9(\mu-C \mathrm{O}), 210.7(\mathrm{CO}), 155.8\left(\mathrm{CO}_{2} \mathrm{Me}\right), 148.9$ (Cipso Xyl), 133.6, 132.8, $132.7\left(C\right.$-Me Xyl $\left.+\mathrm{N}_{3} C_{2}\right), 130.0,128.8,128.7(C \mathrm{H} \mathrm{Xyl}), 88.3,87.2(C p), 54.6,53.7(\mathrm{~N} M e+$ $\left.\mathrm{CO}_{2} M e\right), 39.5\left(\mathrm{~N}_{3} M e\right), 18.7,16.9\left(M_{2} \mathrm{C}_{6} \mathrm{H}_{3}\right)$.

2.10 Synthesis of $\left[\mathrm{M}_{2}\{\mu-\mathrm{CN}(\mathrm{Me})(\mathrm{R})\}(\mu-\mathrm{CO})(\mathrm{CO})\left\{\mathrm{N}_{3}(\mathrm{Me}) \mathrm{C}_{2}(\mathrm{H})\left(\mathrm{CO}_{2} \mathrm{Me}\right)\right\}(\mathrm{Cp})_{2}\right]\left[\mathrm{CF}_{3} \mathrm{SO}_{3}\right](\mathrm{M}=\mathrm{Fe}$, $R=M e, 10 a ; M=R u, R=X y l, 10 b)$.

$\mathrm{CF}_{3} \mathrm{SO}_{3} \mathrm{Me}(0.1 \mathrm{ml}, 0.884 \mathrm{mmol})$ was added to a solution of $7(0.300 \mathrm{mmol})$ in $\mathrm{CH}_{2} \mathrm{Cl}_{2}(6 \mathrm{ml})$; after stirring at room temperature for 30 minutes, the solvent was removed under reduced pressure and the residue washed with $\mathrm{Et}_{2} \mathrm{O}(2 \times 10 \mathrm{ml})$.

10a Yield $172.0 \mathrm{mg}$ (89 \%). Anal. Calcd. For $\mathrm{C}_{21} \mathrm{H}_{23} \mathrm{~F}_{3} \mathrm{Fe}_{2} \mathrm{~N}_{4} \mathrm{O}_{7} \mathrm{~S}$ : C, 39.15; H, 3.60; N, 8.70. Found: C, 39.03; H, 3.82; N, 8.46. IR ( $\left.\mathrm{CH}_{2} \mathrm{Cl}_{2}, 293 \mathrm{~K}\right)$ : v(CO) 1983 (vs), 1799 (s); v(CO $\left.\mathrm{CO}_{2} \mathrm{Me}\right) 1742$ (m), $1718(\mathrm{~m}) ; \gamma(\mu-\mathrm{CN}) 1581(\mathrm{~m}) \mathrm{cm}^{-1} .{ }^{1} \mathrm{H}$ NMR $\left(\mathrm{CDCl}_{3}, 293 \mathrm{~K}\right): \delta 8.03(\mathrm{~s}, 1 \mathrm{H}, \mathrm{CH}), 4.96,4.79$ (s, $10 \mathrm{H}, C p), 4.82,4.30$ (s, 6H, NMe 2 ), 4.03 (s, 3H, $\left.\mathrm{N}_{3} M e\right), 3.84$ (s, 3H, $\mathrm{CO}_{2} \mathrm{Me}$ ).

$10 b$ Yield $235.0 \mathrm{mg}$ (95 \%). Anal. Calcd. For $\mathrm{C}_{28} \mathrm{H}_{29} \mathrm{~F}_{3} \mathrm{~N}_{4} \mathrm{O}_{7} \mathrm{Ru}_{2} \mathrm{~S}$ : C, 40.78; H, 3.54; N, 6.80. Found: C, 40.46; H, 3.74; N, 7.01. IR ( $\left.\mathrm{CH}_{2} \mathrm{Cl}_{2}, 293 \mathrm{~K}\right)$ : v(CO) 1978 (vs), 1806 (s); v( $\left.\mathrm{CO}_{2} \mathrm{Me}\right) 1746$ $(\mathrm{s}) ; \gamma(\mu-\mathrm{CN}) 1528(\mathrm{~ms}) \mathrm{cm}^{-1} .{ }^{1} \mathrm{H}$ NMR $\left(\mathrm{CDCl}_{3}, 293 \mathrm{~K}\right): \delta 8.14(\mathrm{~s}, 1 \mathrm{H}, \mathrm{CH}), 7.24-7.21(\mathrm{~m}, 3 \mathrm{H}$, $\left.\mathrm{Me}_{2} \mathrm{C}_{6} H_{3}\right), 5.34,4.89$ (s, 10H, Cp), 4.36 (s, 3H, NMe), 4.19 (s, 3H, N 3 Me), 3.90 (s, 3H, $\left.\mathrm{CO}_{2} M e\right)$, 2.41, 2.19 (s, 6H, $\left.M e_{2} \mathrm{C}_{6} \mathrm{H}_{3}\right) .{ }^{13} \mathrm{C}\left\{{ }^{1} \mathrm{H}\right\} \mathrm{NMR}\left(\mathrm{CDCl}_{3}, 293 \mathrm{~K}\right): \delta 310.8(\mu-C \mathrm{~N}), 240.0(\mu-C \mathrm{O}), 200.3$ (CO), $156.9\left(\mathrm{CO}_{2} \mathrm{Me}\right), 148.2$ (Cipso Xyl), $143.5(C \mathrm{H}), 130.0,132.6$ (C-Me Xyl), 129.9 $\left(\mathrm{N}_{3} \mathrm{CHCCO}_{2} \mathrm{Me}\right), 129.8,128.8,128.7$ ( $\left.\mathrm{CH} \mathrm{Xyl}\right), 89.6,88.3(C p), 53.3(\mathrm{NMe}), 53.1\left(\mathrm{CO}_{2} \mathrm{Me}\right), 38.7$ $\left(\mathrm{N}_{3} \mathrm{Me}\right), 18.4,16.9\left(\mathrm{Me}_{2} \mathrm{C}_{6} \mathrm{H}_{3}\right)$. 


\subsection{Synthesis of $\left[\mathrm{Fe}_{2}\{\mu-\mathrm{CN}(\mathrm{Me})(\mathrm{Xyl})\}(\mu-\mathrm{CO})(\mathrm{CO})\left\{\mathrm{N}_{3}(\mathrm{Me}) \mathrm{C}_{2}(\mathrm{H})(\mathrm{CN})\right\}(\mathrm{Cp})_{2}\right]\left[\mathrm{CF}_{3} \mathrm{SO}_{3}\right], 11$.}

$\mathrm{CF}_{3} \mathrm{SO}_{3} \mathrm{Me}(0.1 \mathrm{ml}, 0.884 \mathrm{mmol})$ was added to a solution of $\mathbf{8}(190.0 \mathrm{mg}, 0.354 \mathrm{mmol})$ in $\mathrm{CH}_{2} \mathrm{Cl}_{2}$ $(6 \mathrm{ml})$; after stirring at room temperature for 30 minutes, the solvent was removed under reduced pressure and the residue washed with $\mathrm{Et}_{2} \mathrm{O}(2 \times 10 \mathrm{ml})$.

11 Yield $223.5 \mathrm{mg}$ (92 \%). Anal. Calcd. For $\mathrm{C}_{27} \mathrm{H}_{26} \mathrm{~F}_{3} \mathrm{Fe}_{2} \mathrm{~N}_{5} \mathrm{O}_{5} \mathrm{~S}$ : C, 47.26; H, 3.82; N, 10.21 . Found: C, 47.62; H, 3.67; N, 10.45. IR $\left(\mathrm{CH}_{2} \mathrm{Cl}_{2}, 293 \mathrm{~K}\right): v(\mathrm{CN}) 2220(\mathrm{~m}), v(\mathrm{CO}) 1986$ (vs), 1818 $(\mathrm{s}) ; \gamma(\mu-\mathrm{CN}) 1522(\mathrm{~m}) \mathrm{cm}^{-1} .{ }^{1} \mathrm{H}$ NMR $\left(\mathrm{CDCl}_{3}, 293 \mathrm{~K}\right)$ Major isomer: $\delta 9.13(\mathrm{~s}, 1 \mathrm{H}, \mathrm{CH}), 7.30(\mathrm{~m}$, $\left.3 \mathrm{H}, \mathrm{Me}_{2} \mathrm{C}_{6} H_{3}\right), 4.19$ (s, 3H, NMe), 5.07, 4.50 (s, 10H, Cp), 3.85 (s, 3H, N $\left.{ }_{3} M e\right), 2.69,2.03$ (s, 6H, $\left.M e_{2} \mathrm{C}_{6} \mathrm{H}_{3}\right)$; Minor isomer: $\delta 8.37(\mathrm{~s}, 1 \mathrm{H}, \mathrm{CH}), 7.30\left(\mathrm{~m}, 3 \mathrm{H}, \mathrm{Me}_{2} \mathrm{C}_{6} \mathrm{H}_{3}\right), 5.14(\mathrm{~s}, 3 \mathrm{H}, \mathrm{NMe}), 4.98,4.38$ $(\mathrm{s}, 10 \mathrm{H}, C p), 4.34\left(\mathrm{~s}, 3 \mathrm{H}, \mathrm{N}_{3} M e\right), 2.66,2.11\left(\mathrm{~s}, 6 \mathrm{H}, M e_{2} \mathrm{C}_{6} \mathrm{H}_{3}\right)$. Major/Minor $=1.2 .{ }^{13} \mathrm{C}\left\{{ }^{1} \mathrm{H}\right\} \mathrm{NMR}$ $\left(\mathrm{CDCl}_{3}, 293 \mathrm{~K}\right)$ Major isomer: $\delta 336.1(\mu-C \mathrm{~N}), 265.6(\mu-C \mathrm{O}), 210.7(C \mathrm{O}), 148.3$ (Cipso Xyl), 134.8, 132.6 (C-Me Xyl), $132.3\left(\mathrm{~N}_{3} \mathrm{CHCCN}\right), 130.1-128.8(C \mathrm{H} \mathrm{Xyl}), 118.7(C \mathrm{H}), 117.7(C \mathrm{~N})$, 88.2, $86.8(C p), 54.2(\mathrm{NMe}), 41.0\left(\mathrm{~N}_{3} M e\right), 18.3,17.0\left(M_{2} \mathrm{C}_{6} \mathrm{H}_{3}\right)$; Minor isomer: $\delta 338.3(\mu-C \mathrm{~N})$, 261.7 ( $\mu$-CO), 210.7 (CO), 147.7 (Cipso Xyl), 136.7, 132.8 (C-Me Xyl), 131.8 ( $\left.\mathrm{N}_{3} \mathrm{CHCCN}\right), 130.1$ $128.8(C \mathrm{H} \mathrm{Xyl}), 119.2(C \mathrm{H}), 116.8(C \mathrm{~N}), 88.3,88.2(C p), 54.0(\mathrm{NMe}), 40.3\left(\mathrm{~N}_{3} M e\right), 18.5,16.9$ $\left(\mathrm{Me}_{2} \mathrm{C}_{6} \mathrm{H}_{3}\right)$.

\subsection{Crystallography}

Compounds trans-2b, $\mathbf{4 b} \cdot \mathbf{C H}_{2} \mathbf{C l}_{2}, 5 \mathbf{a}, \mathbf{6 b} \cdot \mathbf{0 . 5 C H _ { 2 }} \mathbf{C l}_{2}$ and $\mathbf{8} \cdot \mathbf{C H}_{2} \mathbf{C l}_{2}$ were crystallized from $\mathrm{CH}_{2} \mathrm{Cl}_{2} /$ petroleum ether at $-20{ }^{\circ} \mathrm{C}$. Crystal data were collected on a Bruker AXS SMART 2000 CCD diffractometer using Mo-K $\alpha$ radiation. Intensity data were measured over full diffraction spheres using $0.3^{\circ}$ wide $\omega$ scans, crystal-to-detector distance $5.2 \mathrm{~cm}$. Cell dimensions and orientation matrixes were initially determined from least-squares refinements on reflections measured in 3 sets of 20 exposures collected in three different $\omega$ regions and eventually refined against all reflections. The software SMART [13] was used for collecting frames of data, indexing reflections and determinations of lattice parameters. The collected frames were then processed for integration by the software SAINT and empirical absorption corrections were applied with SADABS [14]. The structure was solved by direct methods and refined by full-matrix least-squares based on all data using $F^{2}$ [15]. Crystal data are listed in Table 1. Non-H atoms were refined anisotropically, unless otherwise stated. H-atoms were placed in calculated positions, and treated isotropically using the 1.2 fold $U_{\text {iso }}$ value of the parent atom except methyl protons, which were assigned the 1.5 fold $U_{\text {iso }}$ value of the parent C-atoms. One $\mathrm{Cp}$ ligand in trans-3b and the $\mathrm{CH}_{2} \mathrm{Cl}_{2}$ molecule in $\mathbf{4 b} \cdot \mathbf{C H}_{2} \mathbf{C l}_{2}$ are disordered. Disordered atomic positions were split and refined 
isotropically using similar distance and similar $U$ restraints and one occupancy parameter per disordered group.

\section{Table 1}

Crystal data and experimental details.

\begin{tabular}{|c|c|c|c|c|c|}
\hline Complex & trans-2b & $4 \mathrm{bb} \cdot \mathrm{CH}_{2} \mathrm{Cl}_{2}$ & $5 \mathbf{a}$ & $6 \mathrm{~b} \cdot 0.5 \mathrm{CH}_{2} \mathrm{Cl}_{2}$ & $8 \cdot \mathrm{CH}_{2} \mathrm{Cl}_{2}$ \\
\hline Formula & $\mathrm{C}_{16} \mathrm{H}_{16} \mathrm{Fe}_{2} \mathrm{~N}_{2} \mathrm{O}_{2} \mathrm{~S}$ & $\mathrm{C}_{24} \mathrm{H}_{24} \mathrm{Cl}_{2} \mathrm{~N}_{2} \mathrm{O}_{3} \mathrm{Ru}_{2}$ & $\mathrm{C}_{22} \mathrm{H}_{22} \mathrm{Fe}_{2} \mathrm{~N}_{4} \mathrm{O}_{2}$ & $\begin{array}{c}\mathrm{C}_{21.5} \mathrm{H}_{23} \mathrm{ClFe}_{2} \mathrm{~N} \\
{ }_{4} \mathrm{O}_{6}\end{array}$ & $\begin{array}{c}\mathrm{C}_{26} \mathrm{H}_{25} \mathrm{Cl}_{2} \mathrm{Fe}_{2} \mathrm{~N}_{5} \\
\mathrm{O}_{2}\end{array}$ \\
\hline$F w$ & 412.07 & 661.49 & 486.14 & 580.59 & 622.11 \\
\hline $\mathrm{T}, \mathrm{K}$ & 293(2) & $293(2)$ & 293(2) & 293(2) & $100(2)$ \\
\hline$\lambda, \AA$ & 0.71073 & 0.71073 & 0.71073 & 0.71073 & 0.71073 \\
\hline Crystal system & Orthorhombic & Monoclinic & Monoclinic & Triclinic & Monoclinic \\
\hline Space group & $P b c a$ & $P 2_{1} / \mathrm{n}$ & $P 2_{1} / \mathrm{c}$ & $\overline{P 1}$ & $P 2_{1} / \mathrm{n}$ \\
\hline$a, \AA$ & $13.5828(5)$ & $13.112(3)$ & $9.0971(4)$ & $9.0434(18)$ & $13.370(3)$ \\
\hline$b, \AA$ & $14.7056(6)$ & $13.975(3)$ & $12.1634(5)$ & $14.838(3)$ & $12.149(2)$ \\
\hline$c, \AA$ & $16.8850(7)$ & $13.879(3)$ & $18.6203(8)$ & $18.788(4)$ & $16.050(3)$ \\
\hline$\alpha,{ }^{\circ}$ & 90 & 90 & 90 & $81.12(3)$ & 90 \\
\hline$\beta,{ }^{\circ}$ & 90 & $93.31(3)$ & $93.5230(10)$ & $86.34(3)$ & $96.04(3)$ \\
\hline$\gamma,{ }^{\circ}$ & 90 & 90 & 90 & $73.67(3)$ & 90 \\
\hline $\begin{array}{c}\text { Cell Volume, } \\
\AA^{3}\end{array}$ & $3372.7(2)$ & 2538.9(9) & $2056.47(15)$ & $2389.8(8)$ & 2592.6(9) \\
\hline$Z$ & 8 & 4 & 4 & 4 & 4 \\
\hline$D_{c}, \mathrm{~g} \mathrm{~cm}^{-3}$ & 1.623 & 1.731 & 1.570 & 1.614 & 1.594 \\
\hline$\mu, \mathrm{mm}^{-1}$ & 1.853 & 1.428 & 1.437 & 1.371 & 1.360 \\
\hline $\mathrm{F}(000)$ & 1680 & 1312 & 1000 & 1188 & 1272 \\
\hline $\begin{array}{c}\text { Crystal size, } \\
\text { mm }\end{array}$ & $0.25 \times 0.22 \times 0.14$ & $0.27 \times 0.23 \times 0.16$ & $0.36 \times 0.28 \times 0.16$ & $0.24 \times 0.29 \times 0.13$ & $0.36 \times 0.27 \times 0.18$ \\
\hline$\theta$ limits, ${ }^{\circ}$ & $2.37-26.37$ & $2.07-25.02$ & $2.00-27.48$ & $1.10-25.03$ & $1.89-27.10$ \\
\hline $\begin{array}{l}\text { Reflections } \\
\text { collected }\end{array}$ & 31402 & 21941 & 21992 & 21312 & 26853 \\
\hline $\begin{array}{l}\text { Independent } \\
\text { reflections }\end{array}$ & $\begin{array}{c}3441\left[\mathrm{R}_{\mathrm{int}}=\right. \\
0.0792]\end{array}$ & $\begin{array}{c}4487\left[\mathrm{R}_{\mathrm{int}}=\right. \\
0.0560]\end{array}$ & $\begin{array}{c}4721\left[\mathrm{R}_{\mathrm{int}}=\right. \\
0.0587]\end{array}$ & $\begin{array}{c}8442\left[\mathrm{R}_{\text {int }}=\right. \\
0.0680]\end{array}$ & $\begin{array}{c}5716\left[\mathrm{R}_{\mathrm{int}}=\right. \\
0.0662]\end{array}$ \\
\hline $\begin{array}{c}\text { Data / } \\
\text { restraints / }\end{array}$ & $3441 / 26 / 206$ & 4487 / 11 / 299 & $4721 / 0$ / 274 & $\begin{array}{c}8442 / 159 / \\
630\end{array}$ & 5716 / 1 / 337 \\
\hline
\end{tabular}




\begin{tabular}{|c|c|c|c|c|c|}
\hline parameters & & & & & \\
\hline $\begin{array}{c}\text { Goodness on } \\
\text { fit on } \mathrm{F}^{2}\end{array}$ & 1.050 & 1.055 & 1.053 & 0.930 & 1.063 \\
\hline $\mathrm{R} 1(\mathrm{I}>2 \sigma(\mathrm{I}))$ & 0.0475 & 0.0427 & 0.0429 & 0.0520 & 0.0454 \\
\hline $\begin{array}{c}\text { WR2 (all } \\
\text { data) }\end{array}$ & 0.1402 & 0.1237 & 0.1169 & 0.1302 & 0.1193 \\
\hline $\begin{array}{c}\text { Largest diff. } \\
\text { peak and hole, } \\
\mathrm{e} \AA^{-3}\end{array}$ & $0.755 /-0.600$ & $1.076 /-0.793$ & $0.457 /-0.340$ & $0.603 /-0.482$ & $0.855 /-0.649$ \\
\hline
\end{tabular}

\section{Results and Discussion}

The $\mathrm{MeCN}$ ligand in the $\mu$-aminocarbyne complexes $\left[\mathrm{Fe}_{2}\{\mu-\mathrm{CN}(\mathrm{Me})(\mathrm{R})\}(\mu-\right.$ $\left.\mathrm{CO})(\mathrm{CO})(\mathrm{MeCN})(\mathrm{Cp})_{2}\right]\left[\mathrm{SO}_{3} \mathrm{CF}_{3}\right](\mathrm{R}=\mathrm{Xyl}, \mathbf{1 a} ; \mathrm{Me}, \mathbf{1 b})$ is almost quantitatively replaced by $\mathrm{NCS}^{-}$ after stirring 1 in THF for 3 hours in the presence of an excess of [NBu 4$][\mathrm{SCN}]$ (Scheme 1). The new complexes trans-[Fe $\left.2\{\mu-\mathrm{CN}(\mathrm{Me})(\mathrm{R})\}(\mu-\mathrm{CO})(\mathrm{CO})(\mathrm{NCS})(\mathrm{Cp})_{2}\right] \square(\mathrm{R}=\mathrm{Xyl}$, trans-2a; Me, trans2b) are, hence, obtained in good yields (71-76 \%) after column chromatography, and they have been fully characterised by IR and NMR spectroscopy. 


\section{Scheme 1}

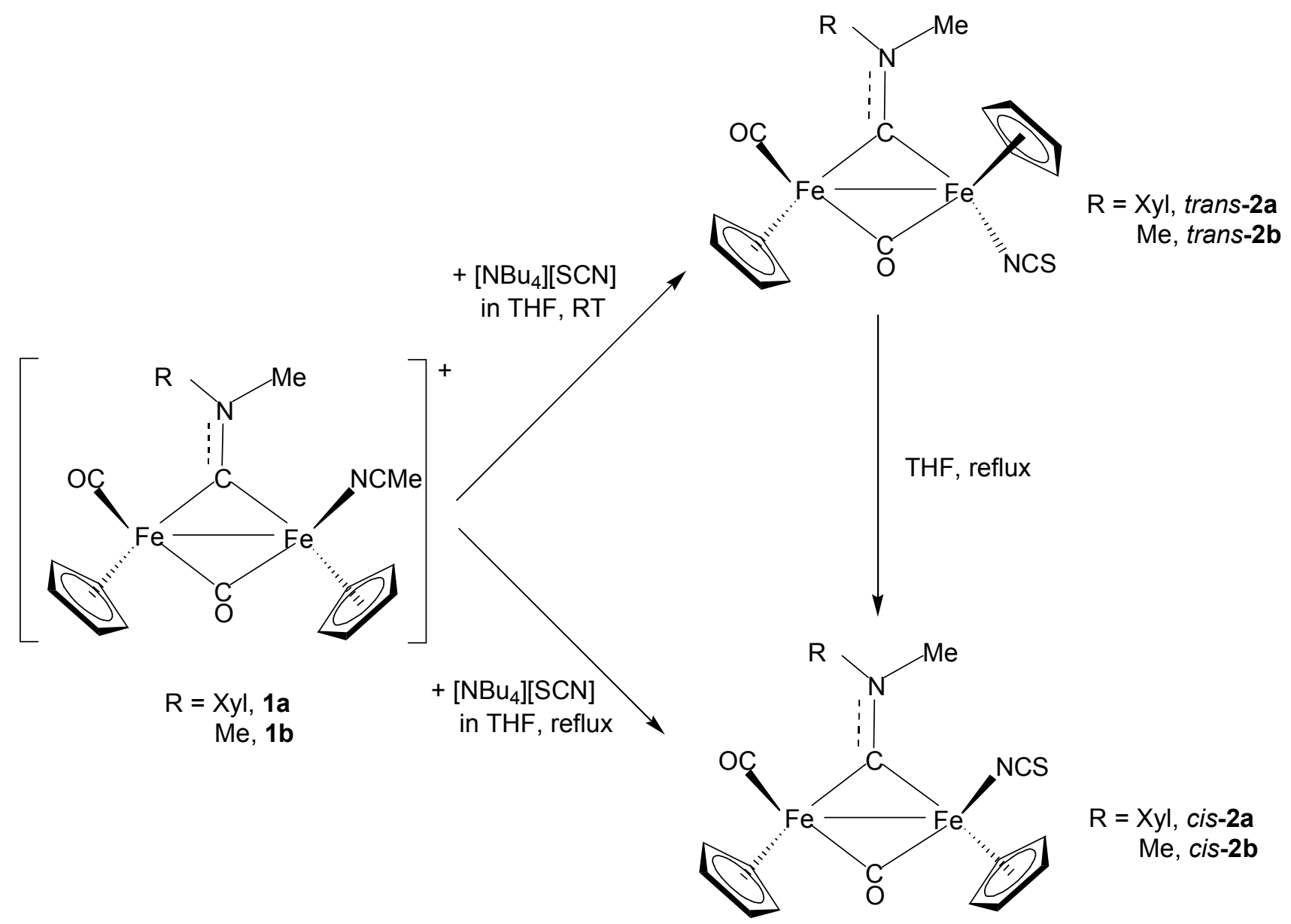

The molecular structure of trans-2b has been, also, determined by X-ray crystallography (Figure 1 and Table 2). The $\mathrm{Cp}$ ligands adopt a trans geometry relative to the mean plane determined by the $\mathrm{Fe}_{2}(\mu-\mathrm{C})_{2}$ core. This is quite unusual, since most of the structures reported for diiron complexes containing a bridging aminocarbyne ligand show a cis geometry of the Cp ligands [8-10]. The $\mathrm{C}(13)-\mathrm{N}(1)$ interaction [1.297(5) $\AA$ ] exhibits a considerably double bond character, suggesting that the $\mu$-aminocarbyne ligand can be alternatively described as a $\mu$-iminium ligand. The $\mathrm{Fe}(1)-\mathrm{N}(2)$ interaction [1.932(3) $\AA$ ] indicates a $\pi$-contribution to the bond; in agreement with this, the $\mathrm{Fe}(1)-\mathrm{N}(2)-\mathrm{C}(16)-\mathrm{S}(1)$ unit is almost linear [bond angles $\mathrm{Fe}(1)-\mathrm{N}(2)-\mathrm{C}(16)$ 169.7(3) and $\left.\mathrm{N}(2)-\mathrm{C}(16)-\mathrm{S}(1) 178.4(4)^{\circ}\right]$ and $\mathrm{N}(2)$ and $\mathrm{C}(16)$ exhibit an almost perfect sp hybridisation. This linear arrangement of the atoms perfectly agrees with a N-coordinated $\mathrm{NCS}^{-}$ligand [16]. Interestingly, the mononuclear complex $\left[\mathrm{Fe}(\mathrm{Cp})(\mathrm{CO})_{2}(\mathrm{NCS})\right]$ exists in both the $\mathrm{N}$ - and S-bonded isomeric forms [17], where the N-bonded isomer is the thermodynamically more stable. The NMR data for trans-2a,b show the presence in solution of only one species, indicating the complete absence of linkage isomerism. 


\section{Figure 1}

Molecular structure of trans-2b, with key atoms labelled (all $\mathrm{H}$ atoms have been omitted).

Displacement ellipsoids are at 30\% probability level.

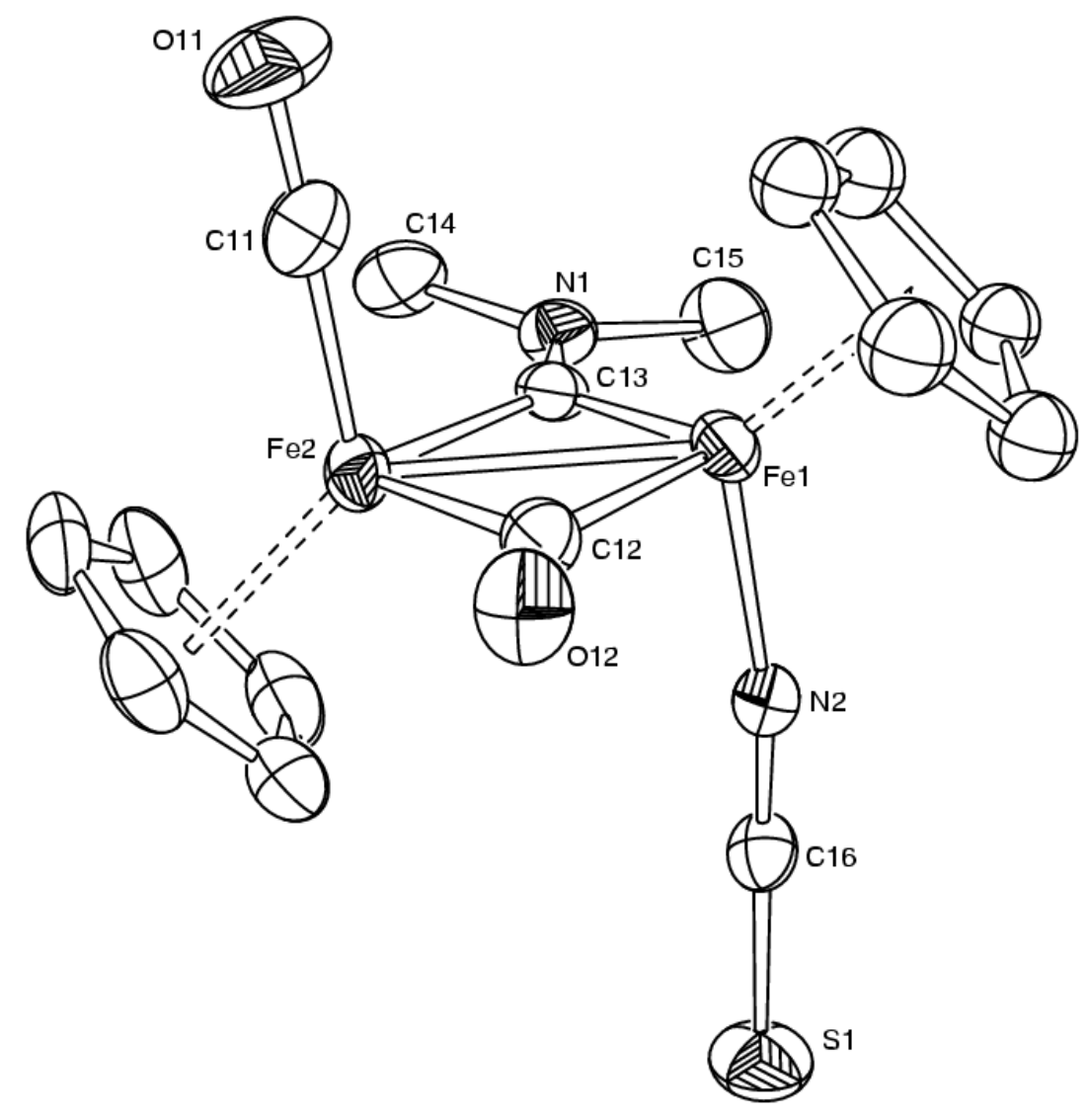

Table 2

Selected bond lengths $(\AA)$ and angles $\left(^{\circ}\right)$ for complex trans-2b.

\begin{tabular}{|c|c|c|c|}
\hline $\mathrm{Fe}(1)-\mathrm{Fe}(2)$ & $2.518(1)$ & $\mathrm{Fe}(1)-\mathrm{N}(2)$ & $1.932(3)$ \\
\hline $\mathrm{Fe}(2)-\mathrm{C}(11)$ & $1.754(5)$ & $\mathrm{N}(2)-\mathrm{C}(16)$ & $1.151(5)$ \\
\hline $\mathrm{Fe}(1)-\mathrm{C}(12)$ & $1.880(4)$ & $\mathrm{C}(16)-\mathrm{S}(1)$ & $1.622(4)$ \\
\hline $\mathrm{Fe}(2)-\mathrm{C}(12)$ & $1.974(5)$ & $\mathrm{C}(11)-\mathrm{O}(11)$ & $1.148(6)$ \\
\hline $\mathrm{Fe}(1)-\mathrm{C}(13)$ & $1.855(4)$ & $\mathrm{C}(12)-\mathrm{O} 12)$ & $1.170(5)$ \\
\hline $\mathrm{Fe}(2)-\mathrm{C}(13)$ & $1.874(4)$ & $\mathrm{C}(13)-\mathrm{N}(1)$ & $1.297(5)$ \\
\hline & & & $169.7(3)$ \\
\hline $\mathrm{Fe}(1)-\mathrm{C}(12)-\mathrm{Fe}(2)$ & $81.55(18)$ & $\mathrm{Fe}(1)-\mathrm{N}(2)-\mathrm{C}(16)$ & $178.4(4)$ \\
\hline $\mathrm{Fe}(1)-\mathrm{C}(13)-\mathrm{Fe}(2)$ & $84.96(17)$ & $\mathrm{N}(2)-\mathrm{C}(16)-\mathrm{S}(1)$ & \\
\hline
\end{tabular}

Complexes trans-2a,b are completely converted into cis-[Fe $2\{\mu-\mathrm{CN}(\mathrm{Me})(\mathrm{R})\}(\mu-$ $\left.\mathrm{CO})(\mathrm{CO})(\mathrm{NCS})(\mathrm{Cp})_{2}\right] \square(\mathrm{R}=\mathrm{Xyl}$, cis-2a; Me, cis-2b) after heating at reflux temperature in THF solution for 5 hours (Scheme 1). The isomerisation is clearly indicated by a shift of $\mathrm{ca} .20 \mathrm{~cm}^{-1}$ 
toward higher frequencies of the terminal $v(\mathrm{CO})$, whereas the other IR bands remain nearly unchanged. Also the NMR data for trans-2 and cis-2 are very similar as expected since they differ only for the relative position of the $\mathrm{Cp}$ ligands. The different arrangement of the $\mathrm{Cp}$ has been further supported by NOE studies; thus, NOE is mutually generated between the two Cp ligans in cis-2 but not in trans-2. Complexes cis-2 can be directly obtained from $\mathbf{1}$ by heating at reflux the latter in the presence of $\mathrm{NCS}^{-}$.

Complex cis-2a reacts with $\mathrm{CF}_{3} \mathrm{SO}_{3} \mathrm{Me}$ to give $c i s-\left[\mathrm{Fe}_{2}\{\mu-\mathrm{CN}(\mathrm{Me})(\mathrm{Xyl})\}(\mu-\right.$ $\left.\mathrm{CO})(\mathrm{CO})(\mathrm{NCSMe})(\mathrm{Cp})_{2}\right]\left[\mathrm{CF}_{3} \mathrm{SO}_{3}\right], 3$ (Scheme 2), as clearly indicated by the spectroscopic data (see Experimental). Addition of lithium acetylides LiCCR $(\mathrm{R}=\mathrm{Me}$, Tol) to 3 results in the elimination of $\mathrm{S}(\mathrm{Me})(\mathrm{CCR})$ with consequent formation of the known compound $c i s-\left[\mathrm{Fe}_{2}\{\mu-\right.$ $\left.\mathrm{CN}(\mathrm{Me})(\mathrm{Xyl})\}(\mu-\mathrm{CO})(\mathrm{CO})(\mathrm{CN})(\mathrm{Cp})_{2}\right]$, in about $80 \%$ yield [9b].

\section{Scheme 2}



Similarly to what reported for the preparation of 2 , the complexes cis- $\left[\mathrm{M}_{2}\{\mu-\right.$ $\left.\mathrm{CN}(\mathrm{Me})(\mathrm{R})\}(\mu-\mathrm{CO})(\mathrm{CO})(\mathrm{NCO})(\mathrm{Cp})_{2}\right] \square(\mathrm{M}=\mathrm{Fe}, \mathrm{R}=\mathrm{Me}, \mathbf{4 a} ; \mathrm{M}=\mathrm{Ru}, \mathrm{R}=\mathrm{Xyl}, \mathbf{4 b} ; \mathrm{M}=\mathrm{Ru}, \mathrm{R}=$ $\mathrm{Me}, \mathbf{4 c})$ and $c i s-\left[\mathrm{M}_{2}\{\mu-\mathrm{CN}(\mathrm{Me})(\mathrm{R})\}(\mu-\mathrm{CO})(\mathrm{CO})\left(\mathrm{N}_{3}\right)(\mathrm{Cp})_{2}\right] \square(\mathrm{M}=\mathrm{Fe}, \mathrm{R}=\mathrm{Xyl}, \mathbf{5 a} ; \mathrm{M}=\mathrm{Fe}, \mathrm{R}=$ $\mathrm{Me}, \mathbf{5 b} ; \mathrm{M}=\mathrm{Ru}, \mathrm{R}=\mathrm{Xyl}, \mathbf{5 c})$ are obtained by heating at reflux temperature $\left[\mathrm{M}_{2}\{\mu-\mathrm{CN}(\mathrm{Me})(\mathrm{R})\}(\mu-\right.$ $\left.\mathrm{CO})(\mathrm{CO})(\mathrm{MeCN})(\mathrm{Cp})_{2}\right]\left[\mathrm{SO}_{3} \mathrm{CF}_{3}\right](\mathrm{M}=\mathrm{Fe}, \mathrm{R}=\mathrm{Xyl}, \mathbf{1 a} ; \mathrm{M}=\mathrm{Fe}, \mathrm{Me}, \mathbf{1 b} ; \mathrm{M}=\mathrm{Ru}, \mathrm{R}=\mathrm{Xyl}, \mathbf{1} \mathbf{c} ; \mathrm{M}=$ $\mathrm{Ru}, \mathrm{R}=\mathrm{Me}, \mathbf{1 d}$ ) in THF in the presence of $\mathrm{NaNCO}$ and $\mathrm{NaN}_{3}$, respectively (Scheme 3). Complexes 4-5 are obtained in good yields (65-76\%) after column chromatography. 


\section{Scheme 3}
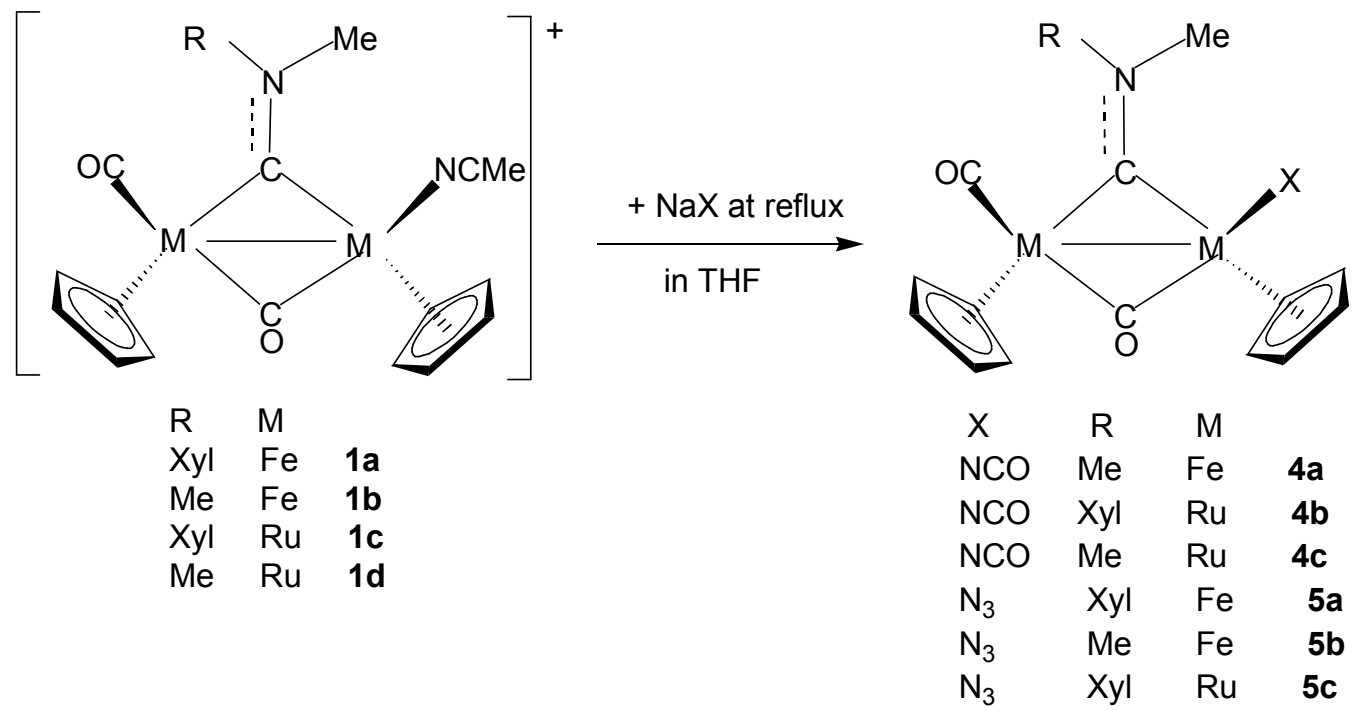

The molecular structures of $\mathbf{4 b}$ and $\mathbf{5 a}$ are reported in Figures 2 and 3, whereas the main bond lengths and bond angles are reported in Tables 3 and 4 . The Cp ligands show in both the molecules a cis arrangement relative to the $\mathrm{M}_{2}(\mu-\mathrm{C})_{2}$ core; moreover, the Xyl substituents in the $\mu$ $\mathrm{CN}(\mathrm{Me})(\mathrm{Xyl})$ ligand points towards the terminal $\mathrm{CO}$ in both $\mathbf{4 b}$ and $\mathbf{5 a}$, as previously found in analogous diiron and diruthenium complexes [10c, 18]. The metal-isocyanate linkage in $\mathbf{4 b}$ is approximately linear [bond angles $\mathrm{Ru}(1)-\mathrm{N}(2)-\mathrm{C}(23) 167.0(5)$ and $\mathrm{N}(2)-\mathrm{C}(23)-\mathrm{O}(2) 177.8(8)^{\circ}$ ], and the $\mathrm{C}(23)-\mathrm{N}(2)$ distance $[1.138(8) \AA]$ is significantly shorter than $\mathrm{C}(23)-\mathrm{O}(2)$ [1.213(8) $\AA]$, implying some triple bond character for the former. This is a clear indication of the $\mathrm{N}$-coordination of the $\mathrm{NCO}^{-}$ligand, as previously demonstrated in complexes such as $\left[\mathrm{Cr}(\mathrm{Cp})(\mathrm{NCO})(\mathrm{NO})_{2}\right][19]$ and $\left[\mathrm{M}(\mathrm{Cp})_{2}(\mathrm{NCO})_{2}\right](\mathrm{M}=\mathrm{Ti}, \mathrm{Zr})$ [5]. Conversely, the azido ligand in 5a shows a bent coordination [bond angle $\left.\mathrm{Fe}(1)-\mathrm{N}(2)-\mathrm{N}(3) 120.1(3)^{\circ}\right]$ and the $\mathrm{Fe}(1)-\mathrm{N}(2)$ distance $[2.009(3) \AA]$ is characteristic for a $\sigma$-interaction, as found in the analogous amino complex $\left[\mathrm{Fe}_{2}\left\{\mu-\mathrm{CN}(\mathrm{Me})_{2}\right\}(\mu-\right.$ $\left.\mathrm{CO})(\mathrm{CO})\left(\mathrm{EtNH}_{2}\right)(\mathrm{Cp})_{2}\right]\left[\mathrm{SO}_{3} \mathrm{CF}_{3}\right][\mathrm{Fe}-\mathrm{N} 2.018(9) \AA][18]$. 


\section{Figure 2}

Molecular structure of $\mathbf{4 b}$, with key atoms labelled (all $\mathrm{H}$ atoms have been omitted). Displacement ellipsoids are at $30 \%$ probability level.

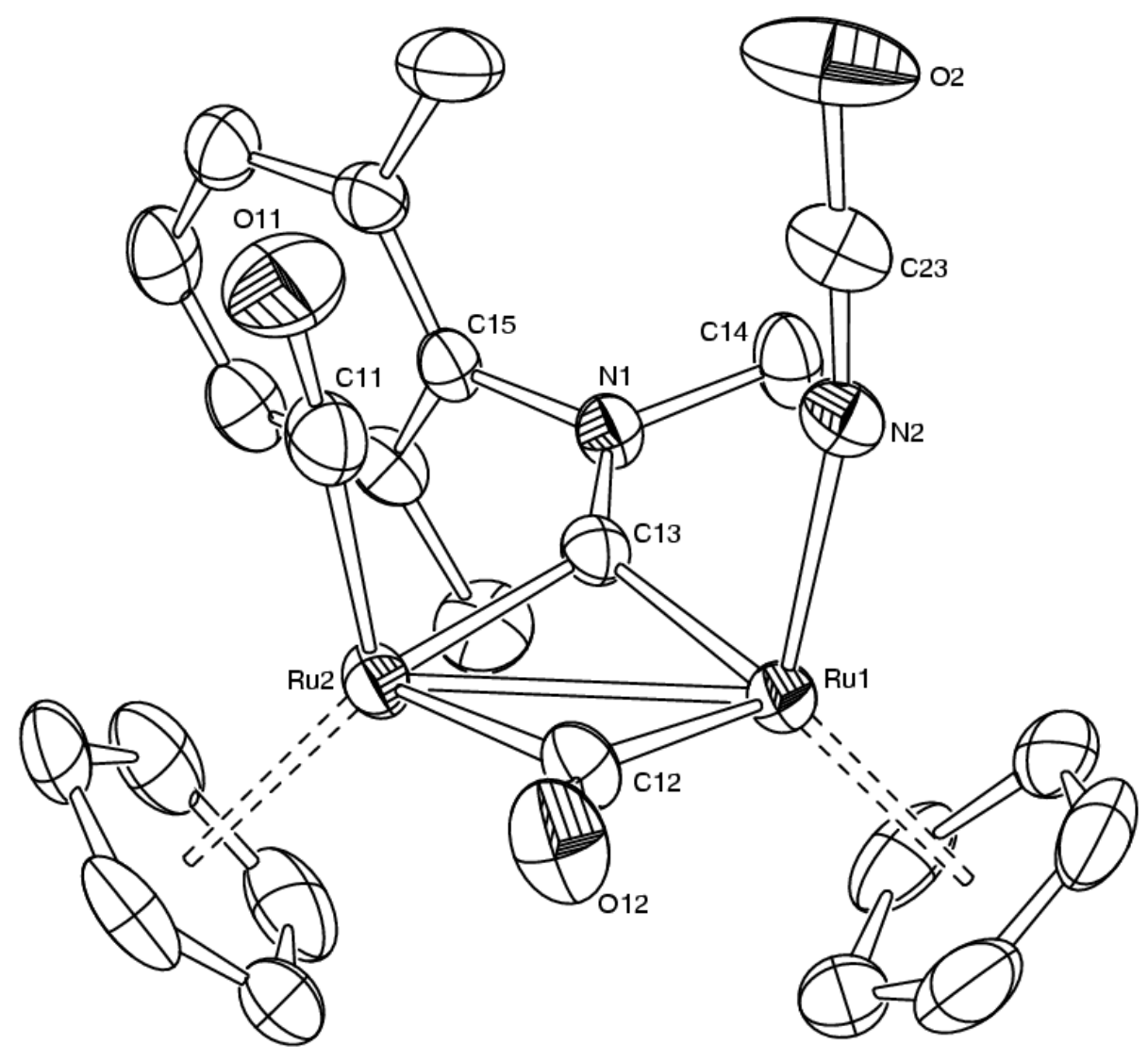

Table 3

Selected bond lengths $(\AA)$ and angles $\left(^{\circ}\right)$ for complex $\mathbf{4 b}$.

\begin{tabular}{|c|c|c|c|}
\hline $\mathrm{Ru}(1)-\mathrm{Ru}(2)$ & $2.7005(8)$ & $\mathrm{Ru}(1)-\mathrm{N}(2)$ & $2.088(5)$ \\
\hline $\mathrm{Ru}(2)-\mathrm{C}(11)$ & $1.850(6)$ & $\mathrm{N}(2)-\mathrm{C}(23)$ & $1.138(8)$ \\
\hline $\mathrm{Ru}(1)-\mathrm{C}(12)$ & $1.981(6)$ & $\mathrm{C}(23)-\mathrm{O}(2)$ & $1.213(8)$ \\
\hline $\mathrm{Ru}(2)-\mathrm{C}(12)$ & $2.108(5)$ & $\mathrm{C}(11)-\mathrm{O}(11)$ & $1.143(7)$ \\
\hline $\mathrm{Ru}(1)-\mathrm{C}(13)$ & $1.940(5)$ & $\mathrm{C}(12)-\mathrm{O} 12)$ & $1.166(7)$ \\
\hline $\mathrm{Ru}(2)-\mathrm{C}(13)$ & $1.991(5)$ & $\mathrm{C}(13)-\mathrm{N}(1)$ & $1.304(6)$ \\
\hline & & & $167.0(35)$ \\
\hline $\mathrm{Fe}(1)-\mathrm{C}(12)-\mathrm{Fe}(2)$ & $82.6(2)$ & $\mathrm{Fe}(1)-\mathrm{N}(2)-\mathrm{C}(16)$ & $177.8(8)$ \\
\hline $\mathrm{Fe}(1)-\mathrm{C}(13)-\mathrm{Fe}(2)$ & $86.8(2)$ & $\mathrm{N}(2)-\mathrm{C}(16)-\mathrm{S}(1)$ & \\
\hline
\end{tabular}




\section{Figure 3}

Molecular structure of 5a, with key atoms labelled (all $\mathrm{H}$ atoms have been omitted). Displacement ellipsoids are at $30 \%$ probability level.

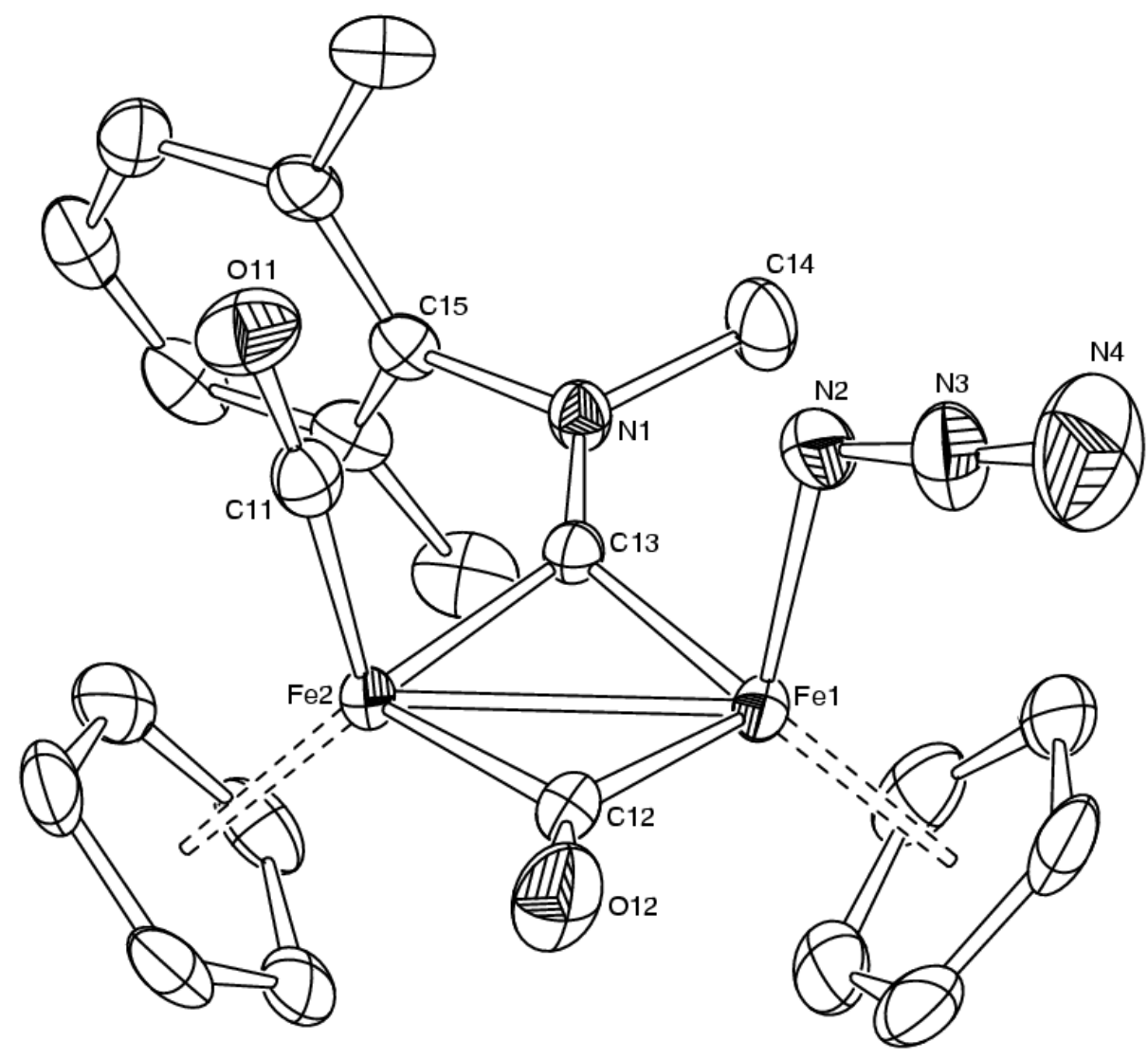

Table 4

Selected bond lengths $(\AA)$ and angles $\left(^{\circ}\right)$ for complex 5a.

\begin{tabular}{|c|c|c|c|}
\hline $\mathrm{Fe}(1)-\mathrm{Fe}(2)$ & $2.4999(6)$ & $\mathrm{Fe}(1)-\mathrm{N}(2)$ & $2.009(3)$ \\
\hline $\mathrm{Fe}(2)-\mathrm{C}(11)$ & $1.753(4)$ & $\mathrm{N}(2)-\mathrm{N}(3)$ & $1.187(4)$ \\
\hline $\mathrm{Fe}(1)-\mathrm{C}(12)$ & $1.882(3)$ & $\mathrm{N}(3)-\mathrm{N}(4)$ & $1.161(5)$ \\
\hline $\mathrm{Fe}(2)-\mathrm{C}(12)$ & $1.968(3)$ & $\mathrm{C}(11)-\mathrm{O}(11)$ & $1.143(4)$ \\
\hline $\mathrm{Fe}(1)-\mathrm{C}(13)$ & $1.849(3)$ & $\mathrm{C}(12)-\mathrm{O} 12)$ & $1.173(4)$ \\
\hline $\mathrm{Fe}(2)-\mathrm{C}(13)$ & $1.877(3)$ & $\mathrm{C}(13)-\mathrm{N}(1)$ & $1.304(4)$ \\
\hline & & & $120.1(3)$ \\
\hline $\mathrm{Fe}(1)-\mathrm{C}(12)-\mathrm{Fe}(2)$ & $80.94(14)$ & $\mathrm{Fe}(1)-\mathrm{N}(2)-\mathrm{C}(16)$ & $176.8(5)$ \\
\hline $\mathrm{Fe}(1)-\mathrm{C}(13)-\mathrm{Fe}(2)$ & $84.29(13)$ & $\mathrm{N}(2)-\mathrm{C}(16)-\mathrm{S}(1)$ & \\
\hline
\end{tabular}

The IR spectra of 4-5 show $v(\mathrm{CO})$ for the terminal and bridging carbonyls at $c a .1980$ and $1800 \mathrm{~cm}^{-1}$, respectively; the former data well agrees with a cis geometry of the Cp ligands also in solution. Moreover, complexes 4 show $v(\mathrm{NCO})$ at $c a .2235 \mathrm{~cm}^{-1}$, whereas $v\left(\mathrm{~N}_{3}\right)$ in $\mathbf{5}$ absorbs at $c a$. 
$2030 \mathrm{~cm}^{-1}$. The NMR spectra of 4-5 show the presence in solution of a single species, except for $\mathbf{5 a}$ where a second isomer is present (major : minor $=10$ ). This is probably due to a different orientation of the Me and $\mathrm{Xyl}$ substituents on the $\mu-\mathrm{CN}(\mathrm{Me})(\mathrm{Xyl})$ ligand (Scheme 4), as previously found in analogous diiron and diruthenium $\mu$-aminocarbyne complexes [10c, 18]. The most important feature of the ${ }^{13} \mathrm{C}$ NMR spectra is the presence of three resonances at low fields attributable to the $\mu$-aminocarbyne carbon $(\delta c a .340 \mathrm{ppm} \mathrm{M}=\mathrm{Fe} ; 310 \mathrm{ppm} \mathrm{M}=\mathrm{Ru})$, the $\mu-\mathrm{CO}(\delta c a$. $266 \mathrm{ppm} \mathrm{M}=\mathrm{Fe} ; 236 \mathrm{ppm} \mathrm{M}=\mathrm{Ru}$ ) and the terminal $\mathrm{CO}$ ligand ( $\delta c a .211 \mathrm{ppm} \mathrm{M}=\mathrm{Fe} ; 200 \mathrm{ppm}$ $\mathrm{M}=\mathrm{Ru}$ ). The carbon of the NCO ligand in $\mathbf{4}$ resonates at $c a .128 \mathrm{ppm}$ as expected for isocyanate ligands.

\section{Scheme 4}

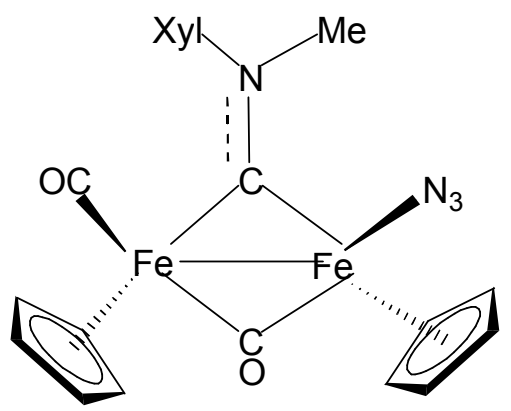

Major $(\alpha)$

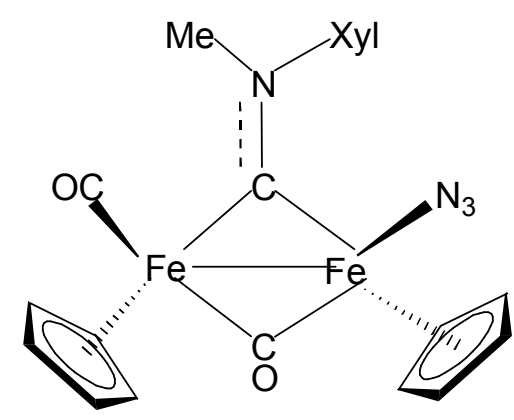

Minor $(\beta)$

The azido complexes 5 react in THF solution at reflux with electron poor alkynes such as $\mathrm{MeO}_{2} \mathrm{CC} \equiv \mathrm{CCO}_{2} \mathrm{Me}$ and $\mathrm{HC} \equiv \mathrm{CCO}_{2} \mathrm{Me}$ affording the triazolato complexes $\left[\mathrm{M}_{2}\{\mu-\mathrm{CN}(\mathrm{Me})(\mathrm{R})\}(\mu-\right.$ $\left.\mathrm{CO})(\mathrm{CO})\left\{\mathrm{N}_{3} \mathrm{C}_{2}\left(\mathrm{CO}_{2} \mathrm{Me}\right)_{2}\right\}(\mathrm{Cp})_{2}\right](\mathrm{M}=\mathrm{Fe}, \mathrm{R}=\mathrm{Xyl}, \mathbf{6 a} ; \mathrm{M}=\mathrm{Fe}, \mathrm{R}=\mathrm{Me}, \mathbf{6 b} ; \mathrm{M}=\mathrm{Ru}, \mathrm{R}=\mathrm{Xyl}, \mathbf{6 c})$ and $\left[\mathrm{M}_{2}\{\mu-\mathrm{CN}(\mathrm{Me})(\mathrm{R})\}(\mu-\mathrm{CO})(\mathrm{CO})\left\{\mathrm{N}_{3} \mathrm{C}_{2}(\mathrm{H})\left(\mathrm{CO}_{2} \mathrm{Me}\right)\right\}(\mathrm{Cp})_{2}\right](\mathrm{M}=\mathrm{Fe}, \mathrm{R}=\mathrm{Me}, 7 \mathbf{a} ; \mathrm{M}=\mathrm{Ru}, \mathrm{R}=$ Xyl, 7b) (Scheme 5). In a similar fashion, the reaction of 5a with fumaronitrile $(\mathrm{NC})(\mathrm{H}) \mathrm{C}=\mathrm{C}(\mathrm{H})(\mathrm{CN})$ under analogous conditions affords the triazolato product $\left[\mathrm{Fe}_{2}\{\mu-\right.$ $\left.\mathrm{CN}(\mathrm{Me})(\mathrm{Xyl})\}(\mu-\mathrm{CO})(\mathrm{CO})\left\{\mathrm{N}_{3} \mathrm{C}_{2}(\mathrm{H})(\mathrm{CN})\right\}(\mathrm{Cp})_{2}\right]$, 8. All these products have been obtained in good yields (70-90 \%) after column chromatography and fully characterised spectroscopically via IR and NMR. Moreover, the molecular structures of $\mathbf{6 b}$ and $\mathbf{8}$ have been determined by X-ray diffraction studies (Figures 4 and 5, and Tables 5 and 6). The formation of 6-8 proceeds via [3+2] cycloaddition of the alkyne or the alkene, as previously demonstrated for mononuclear azido complexes $[7,20]$; in the case of $\mathbf{8}$, the reaction is accompanied by loss of $\mathrm{HCN}$. 


\section{Scheme 5}






\section{Figure 4}

Molecular structure of $\mathbf{6 b}$, with key atoms labelled (all $\mathrm{H}$ atoms have been omitted). Displacement ellipsoids are at 30\% probability level. Only one of the two independent molecules is represented.

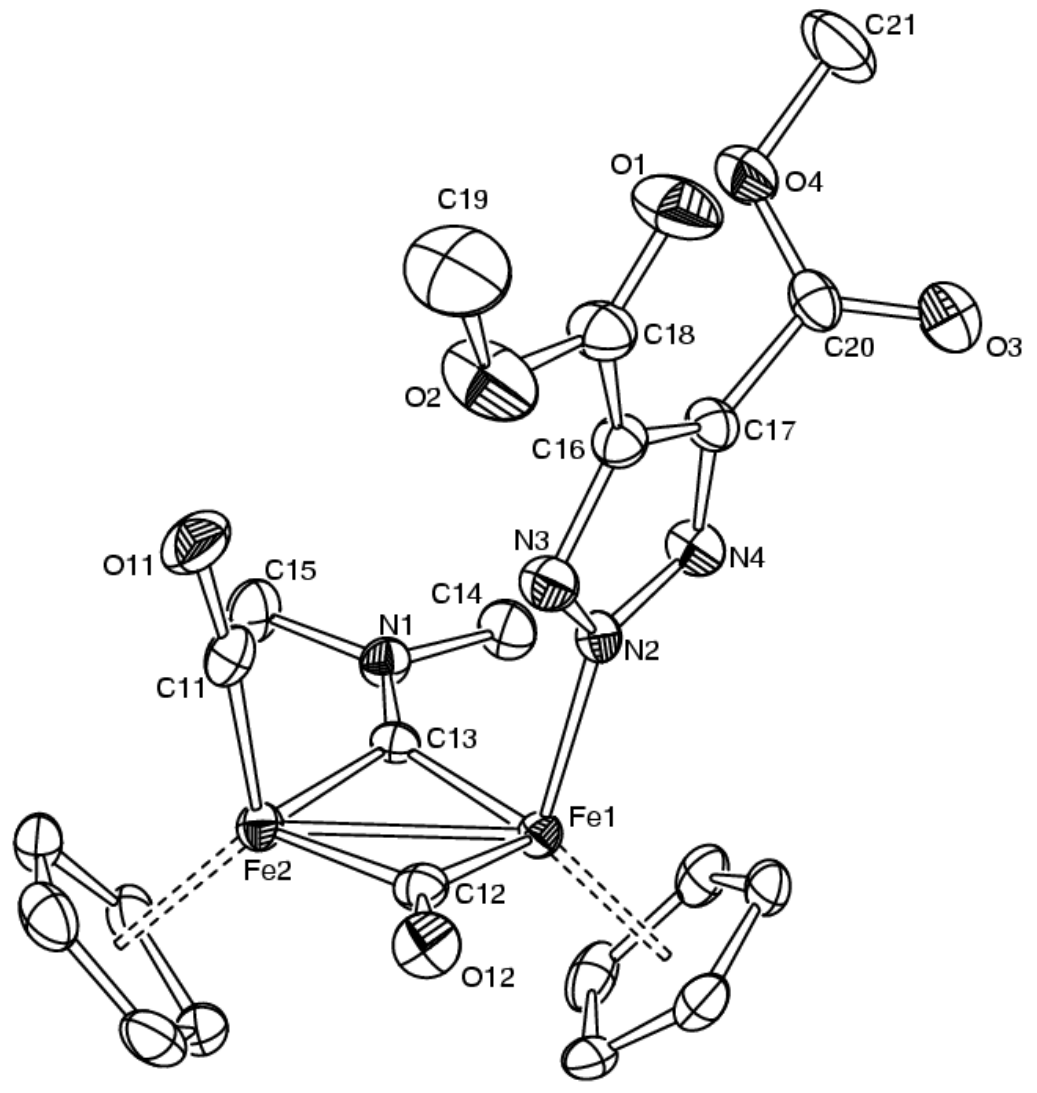

Table 5

Selected bond lengths $(\AA)$ and angles $\left(^{\circ}\right)$ for the two independent molecules of complex $\mathbf{6 b}$.

\begin{tabular}{|c|c|c|c|}
\hline $\mathrm{Fe}(1)-\mathrm{Fe}(2)$ & $2.5200(11)$ & $\mathrm{Fe}(3)-\mathrm{Fe}(4)$ & $2.5062(11)$ \\
\hline $\mathrm{Fe}(2)-\mathrm{C}(11)$ & $1.753(5)$ & $\mathrm{Fe}(4)-\mathrm{C}(41)$ & $1.755(6)$ \\
\hline $\mathrm{Fe}(1)-\mathrm{C}(12)$ & $1.881(5)$ & $\mathrm{Fe}(3)-\mathrm{C}(42)$ & $1.861(5)$ \\
\hline $\mathrm{Fe}(2)-\mathrm{C}(12)$ & $1.968(5)$ & $\mathrm{Fe}(4)-\mathrm{C}(42)$ & $1.969(5)$ \\
\hline $\mathrm{Fe}(1)-\mathrm{C}(13)$ & $1.842(5)$ & $\mathrm{Fe}(3)-\mathrm{C}(43)$ & $1.845(5)$ \\
\hline $\mathrm{Fe}(2)-\mathrm{C}(13)$ & $1.885(4)$ & $\mathrm{Fe}(4)-\mathrm{C}(43)$ & $1.877(5)$ \\
\hline $\mathrm{Fe}(1)-\mathrm{N}(2)$ & $1.998(4)$ & $\mathrm{Fe}(3)-\mathrm{N}(6)$ & $1.989(4)$ \\
\hline $\mathrm{N}(2)-\mathrm{N}(3)$ & $1.318(5)$ & $\mathrm{N}(6)-\mathrm{N}(7)$ & $1.315(5)$ \\
\hline $\mathrm{N}(2)-\mathrm{N}(4)$ & $1.330(5)$ & $\mathrm{N}(6)-\mathrm{N}(8)$ & $1.320(5)$ \\
\hline $\mathrm{N}(3)-\mathrm{C}(16)$ & $1.362(6)$ & $\mathrm{N}(7)-\mathrm{C}(46)$ & $1.369(6)$ \\
\hline $\mathrm{N}(4)-\mathrm{C}(17)$ & $1.347(6)$ & $\mathrm{N}(8)-\mathrm{C}(47)$ & $1.354(6)$ \\
\hline
\end{tabular}




\begin{tabular}{|c|c|c|c|}
\hline $\mathrm{C}(16)-\mathrm{C}(17)$ & $1.391(6)$ & $\mathrm{C}(46)-\mathrm{C}(47)$ & $1.383(6)$ \\
\hline $\mathrm{C}(11)-\mathrm{O}(11)$ & $1.149(6)$ & $\mathrm{C}(41)-\mathrm{O}(41)$ & $1.141(5)$ \\
\hline $\mathrm{C}(12)-\mathrm{O}(12)$ & $1.176(5)$ & $\mathrm{C}(42)-\mathrm{O}(42)$ & $1.178(5)$ \\
\hline $\mathrm{C}(13)-\mathrm{N}(1)$ & $1.305(5)$ & $\mathrm{C}(43)-\mathrm{N}(5)$ & $1.307(6)$ \\
\hline & & & $81.7(2)$ \\
\hline $\mathrm{Fe}(1)-\mathrm{C}(12)-\mathrm{Fe}(2)$ & $81.77(19)$ & $\mathrm{Fe}(3)-\mathrm{C}(42)-\mathrm{Fe}(4)$ & $84.6(2)$ \\
\hline $\mathrm{Fe}(1)-\mathrm{C}(13)-\mathrm{Fe}(2)$ & $85.06(19)$ & $\mathrm{Fe}(3)-\mathrm{C}(43)-\mathrm{Fe}(4)$ & $124.8(3)$ \\
\hline $\mathrm{Fe}(1)-\mathrm{N}(2)-\mathrm{N}(3)$ & $123.0(3)$ & $\mathrm{Fe}(3)-\mathrm{N}(6)-\mathrm{N}(7)$ & $104.9(4)$ \\
\hline $\mathrm{N}(2)-\mathrm{N}(3)-\mathrm{C}(16)$ & $105.0(4)$ & $\mathrm{N}(6)-\mathrm{N}(7)-\mathrm{C}(46)$ & $107.3(4)$ \\
\hline $\mathrm{N}(3)-\mathrm{C}(16)-\mathrm{C}(17)$ & $107.3(4)$ & $\mathrm{N}(7)-\mathrm{C}(46)-\mathrm{C}(47)$ & $108.3(4)$ \\
\hline $\mathrm{C}(16)-\mathrm{C}(17)-\mathrm{N}(4)$ & $108.5(4)$ & $\mathrm{C}(46)-\mathrm{C}(47)-\mathrm{N}(8)$ & $104.8(4)$ \\
\hline $\mathrm{C}(17)-\mathrm{N}(4)-\mathrm{N}(2)$ & $104.7(4)$ & $\mathrm{C}(47)-\mathrm{N}(8)-\mathrm{N}(6)$ & \\
\hline
\end{tabular}

Figure 5

Molecular structure of $\mathbf{8}$, with key atoms labelled (all $\mathrm{H}$ atoms, except $\mathrm{H} 23$, have been omitted). Displacement ellipsoids are at 30\% probability level.

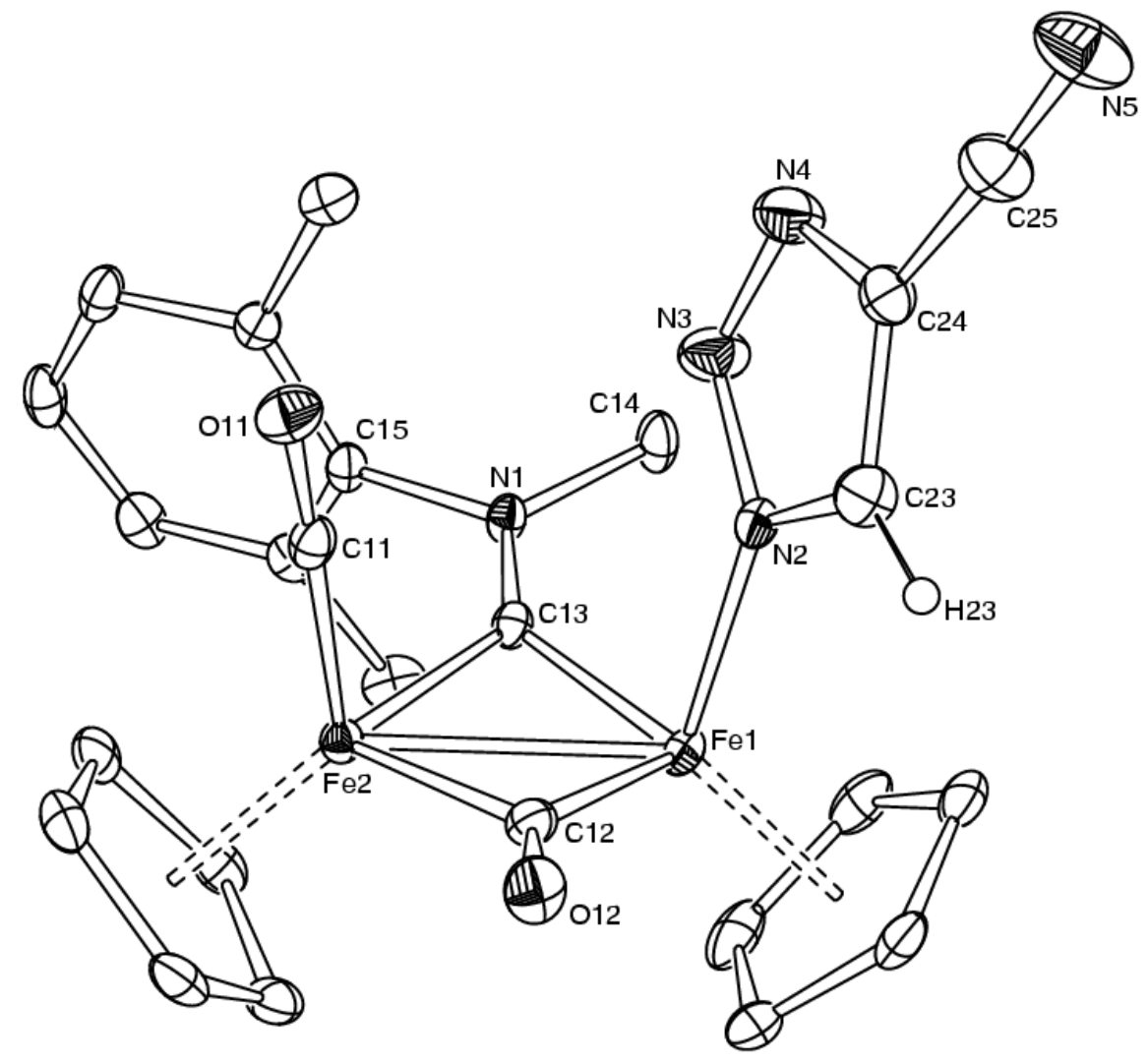


Table 6

Selected bond lengths $(\AA)$ and angles $\left(^{\circ}\right)$ for complex $\mathbf{8}$.

\begin{tabular}{|c|c|c|c|}
\hline $\mathrm{Fe}(1)-\mathrm{Fe}(2)$ & $2.5084(8)$ & & \\
\hline $\mathrm{Fe}(2)-\mathrm{C}(11)$ & $1.760(3)$ & $\mathrm{N}(2)-\mathrm{N}(3)$ & $1.351(2)$ \\
\hline $\mathrm{Fe}(1)-\mathrm{C}(12)$ & $1.880(3)$ & $\mathrm{N}(3)-\mathrm{N}(4)$ & $1.326(4)$ \\
\hline $\mathrm{Fe}(2)-\mathrm{C}(12)$ & $1.977(3)$ & $\mathrm{N}(4)-\mathrm{C}(24)$ & $1.336(4)$ \\
\hline $\mathrm{Fe}(1)-\mathrm{C}(13)$ & $1.863(2)$ & $\mathrm{C}(23)-\mathrm{C}(24)$ & $1.432(4)$ \\
\hline $\mathrm{Fe}(2)-\mathrm{C}(13)$ & $1.892(2)$ & $\mathrm{C}(24)-\mathrm{C}(25)$ & $1.151(4)$ \\
\hline $\mathrm{Fe}(1)-\mathrm{N}(2)$ & $1.970(2)$ & $\mathrm{C}(25)-\mathrm{N}(5)$ & $1.305(3)$ \\
\hline $\mathrm{C}(11)-\mathrm{O}(11)$ & $1.150(3)$ & $\mathrm{C}(13)-\mathrm{N}(1)$ & $1.481(3)$ \\
\hline $\mathrm{C}(12)-\mathrm{O}(12)$ & $1.179(3)$ & $\mathrm{C}(14)-\mathrm{N}(1)$ & $107.1(2)$ \\
\hline $\mathrm{Fe}(1)-\mathrm{C}(12)-\mathrm{Fe}(2)$ & $81.10(10)$ & $\mathrm{N}(3)-\mathrm{N}(4)-\mathrm{C}(24)$ & $108.8(2)$ \\
\hline $\mathrm{Fe}(1)-\mathrm{C}(13)-\mathrm{Fe}(2)$ & $83.83(10)$ & $\mathrm{N}(4)-\mathrm{C}(24)-\mathrm{C}(23)$ & $106.7(2)$ \\
\hline $\mathrm{Fe}(1)-\mathrm{N}(2)-\mathrm{N}(3)$ & $122.66(18)$ & $\mathrm{C}(24)-\mathrm{C}(23)-\mathrm{N}(2)$ & $176.9(4)$ \\
\hline $\mathrm{N}(2)-\mathrm{N}(3)-\mathrm{N}(4)$ & $110.0(2)$ & $\mathrm{C}(24)-\mathrm{C}(25)-\mathrm{N}(5)$ & \\
\hline
\end{tabular}

The asymmetric unit of $\mathbf{6 b}$ contains two independent molecules, with similar relative arrangement of the atoms, similar bond lengths and bond angles but opposite absolute structure. The molecular structures of $\mathbf{6 b}$ and $\mathbf{8}$ exhibit a cis arrangement of the Cp ligands; moreover, in the case of $\mathbf{8}$ where the bridging aminocarbyne ligand is asymmetrically substituted, the bulkier Xyl group points in the direction of the terminal CO ligand. The triazolato ligand is $\mathrm{N}(2)$ coordinated in $\mathbf{6 b}$, whereas it is bound to iron via $\mathrm{N}(1)$ in $\mathbf{8}$. Both these coordination modes of the triazolato ligand have been previously reported [20a]; molecular orbital calculations [21] indicate that these two bonding modes are essentially isoenergetic. Evidence obtained to date indicates that either two isomers $\mathrm{N}(1)$ and $\mathrm{N}(2)$ are formed simultaneously or only the $\mathrm{N}(2)$ isomer is produced exclusively $[7,20 \mathrm{c}, 22]$. Our work completely support this hypothesis; in fact, in the case of complexes 6 which contain a symmetrically disubstituted triazolato ligand $\mathrm{N}_{3} \mathrm{C}_{2}\left(\mathrm{CO}_{2} \mathrm{Me}\right)_{2}$, only the $\mathrm{N}(2)$-bound isomer is formed as shown by NMR data. Conversely, the complexes 7-8, which posses a monosubstituted triazolato ligand, are formed as a mixture of $\mathrm{N}(1)$ and $\mathrm{N}(2)$ isomers (Scheme 6). 


\section{Scheme 6}



$\mathrm{N}(2)$ Isomer

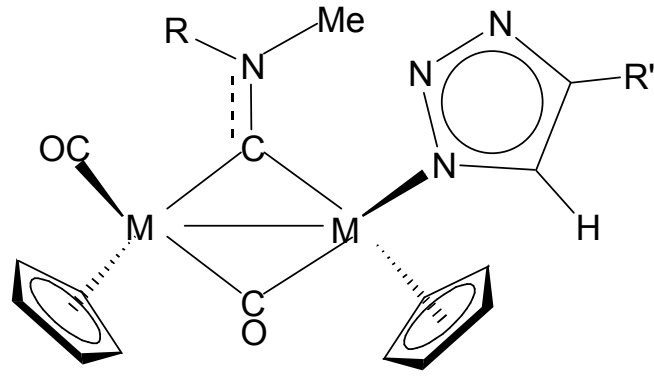

$N(1)$ Isomer

The IR spectra for 6-8 show two $v(\mathrm{CO})$ at frequencies similar to the starting azido complexes 5, indicating a similar electronic character of the two ligands. As a consequence of the symmetrical $\mathrm{N}(2)$ coordination, the carbon atoms of the triazolato ligand and the two COOMe substituents in $\mathbf{6}$ are equivalent and give rise to single NMR resonances [e.g. $\delta_{\mathrm{H}}$ 3.7-3.8 ppm for $\mathrm{CO}_{2} \mathrm{Me} ; \delta_{\mathrm{C}} c a$. 162, 140 and $52 \mathrm{ppm}$ for $\mathrm{N}_{3} \mathrm{C}_{2}\left(\mathrm{CO}_{2} \mathrm{Me}\right)_{2}, \mathrm{~N}_{3} \mathrm{C}_{2}\left(\mathrm{CO}_{2} \mathrm{Me}\right)_{2}$ and $\mathrm{N}_{3} \mathrm{C}_{2}\left(\mathrm{CO}_{2} \mathrm{Me}\right)_{2}$, respectively]. Conversely, for $\mathbf{7 - 8}$ two sets of resonances are present for the $\mathrm{N}(2)$ and $\mathrm{N}(1)$ isomers $[\mathrm{N}(2): \mathrm{N}(1)$ isomer ratio $=1.1-2: 1]$. NOE studies clearly indicate that the major isomer is the $\mathrm{N}(1)$-bound, which is the one also present in the solid state for $\mathbf{8}$. For instance, irradiation of the triazolato proton $\left(\delta_{\mathrm{H}} 7.74 \mathrm{ppm}\right)$ in the major isomer of $\mathbf{8}$ produces a strong enhancement of the resonance of one $\mathrm{Cp}$ ligand, whereas no effect is observed after irradiation of the corresponding resonance in the minor isomer $\left(\delta_{\mathrm{H}} 7.46 \mathrm{ppm}\right)$.

The triazolato complexes 6-7 react in $\mathrm{CH}_{2} \mathrm{Cl}_{2}$ solution with $\mathrm{CF}_{3} \mathrm{SO}_{3} \mathrm{Me}$ affording the $\mathrm{N}$ methylated cationic complexes

$\left[\mathrm{Fe}_{2}\{\mu-\mathrm{CN}(\mathrm{Me})(\mathrm{Xyl})\}(\mu-\right.$ $\left.\mathrm{CO})(\mathrm{CO})\left\{\mathrm{N}_{3}(\mathrm{Me}) \mathrm{C}_{2}\left(\mathrm{CO}_{2} \mathrm{Me}\right)_{2}\right\}(\mathrm{Cp})_{2}\right]\left[\mathrm{CF}_{3} \mathrm{SO}_{3}\right], \quad \mathbf{9}, \quad$ and $\quad\left[\mathrm{M}_{2}\{\mu-\mathrm{CN}(\mathrm{Me})(\mathrm{R})\}(\mu-\right.$ $\left.\mathrm{CO})(\mathrm{CO})\left\{\mathrm{N}_{3}(\mathrm{Me}) \mathrm{C}_{2}(\mathrm{H})\left(\mathrm{CO}_{2} \mathrm{Me}\right)\right\}(\mathrm{Cp})_{2}\right]\left[\mathrm{CF}_{3} \mathrm{SO}_{3}\right](\mathrm{M}=\mathrm{Fe}, \mathrm{R}=\mathrm{Me}, \mathbf{1 0 a} ; \mathrm{M}=\mathrm{Ru}, \mathrm{R}=\mathrm{Xyl}, \mathbf{1 0 b})$, in good yields (90-95\%) (Scheme 7). 


\section{Scheme 7}

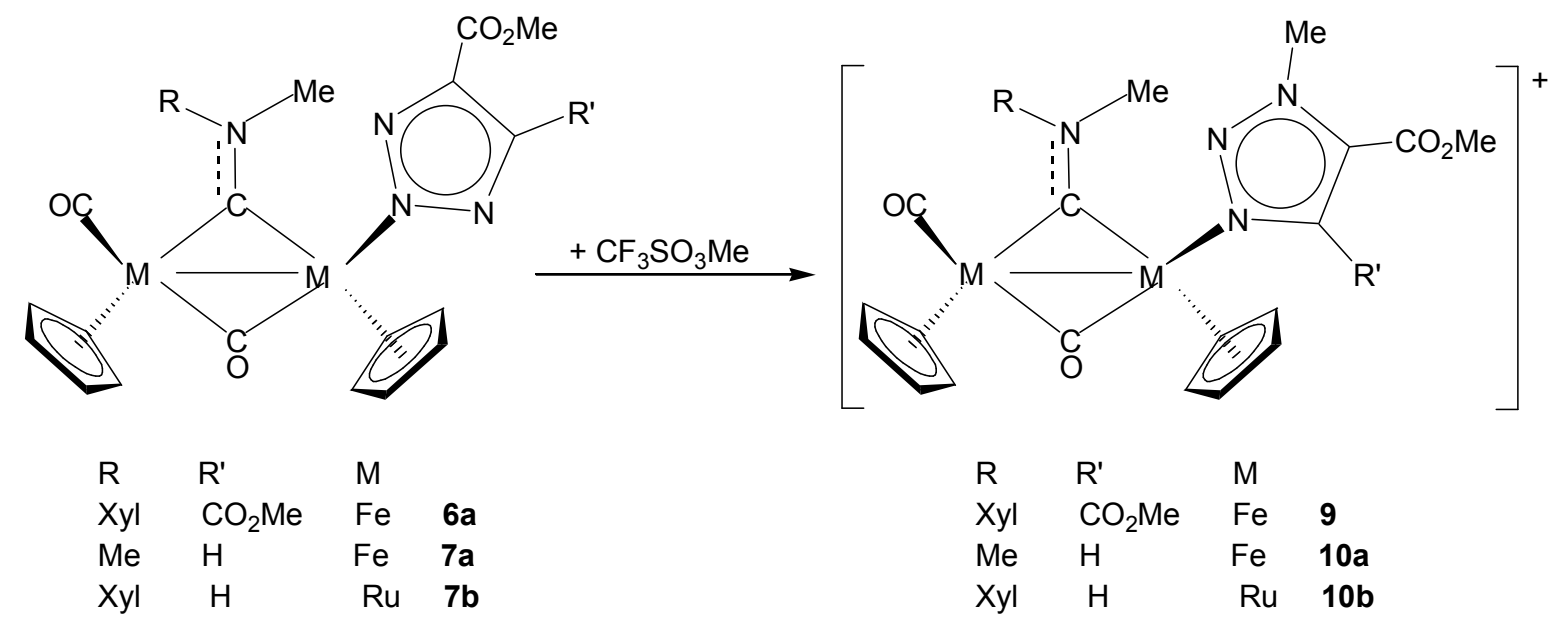

Their NMR spectra show the presence in solution of a single species; NOE experiments on $10 \mathrm{~b}$ clearly indicate a strong interaction between one $\mathrm{Cp}$ ligand and the $\mathrm{CH}$ proton of the triazole ring, suggesting that methylation occurs selectively at N(3) and the triazole is N(1) coordinated. It is interesting to notice that in previous works [7] a similar regiochemistry for the methylation process was observed, but it was, then, followed by the release of the triazole molecule. Conversely, in our case the complexes 9-10 are stable in solution and do not lose the heterocycle ligand. In a similar way, the methylation of $\mathbf{8}$ results in the nearly quantitative formation of the complex $\left[\mathrm{Fe}_{2}\{\mu\right.$ $\left.\mathrm{CN}(\mathrm{Me})(\mathrm{Xyl})\}(\mu-\mathrm{CO})(\mathrm{CO})\left\{\mathrm{N}_{3}(\mathrm{Me}) \mathrm{C}_{2}(\mathrm{H})(\mathrm{CN})\right\}(\mathrm{Cp})_{2}\right]\left[\mathrm{CF}_{3} \mathrm{SO}_{3}\right]$, 11. The IR spectrum shows the usual $v(\mathrm{CO})$ for the terminal and bridging carbonyls at frequencies similar to those observed for 910, and $v(\mathrm{CN})$ at $2220 \mathrm{~cm}^{-1}$; hence, also in this case methylation has occurred on the triazolato ring. The NMR spectra show the presence of two isomers in a 1.2:1 ratio and with very similar but distinct resonances. This can be explained assuming that the methylation of $\mathbf{8}$ is not regioselective, but it can occur on both N(1) and N(3) (Scheme 8). 


\section{Scheme 8}
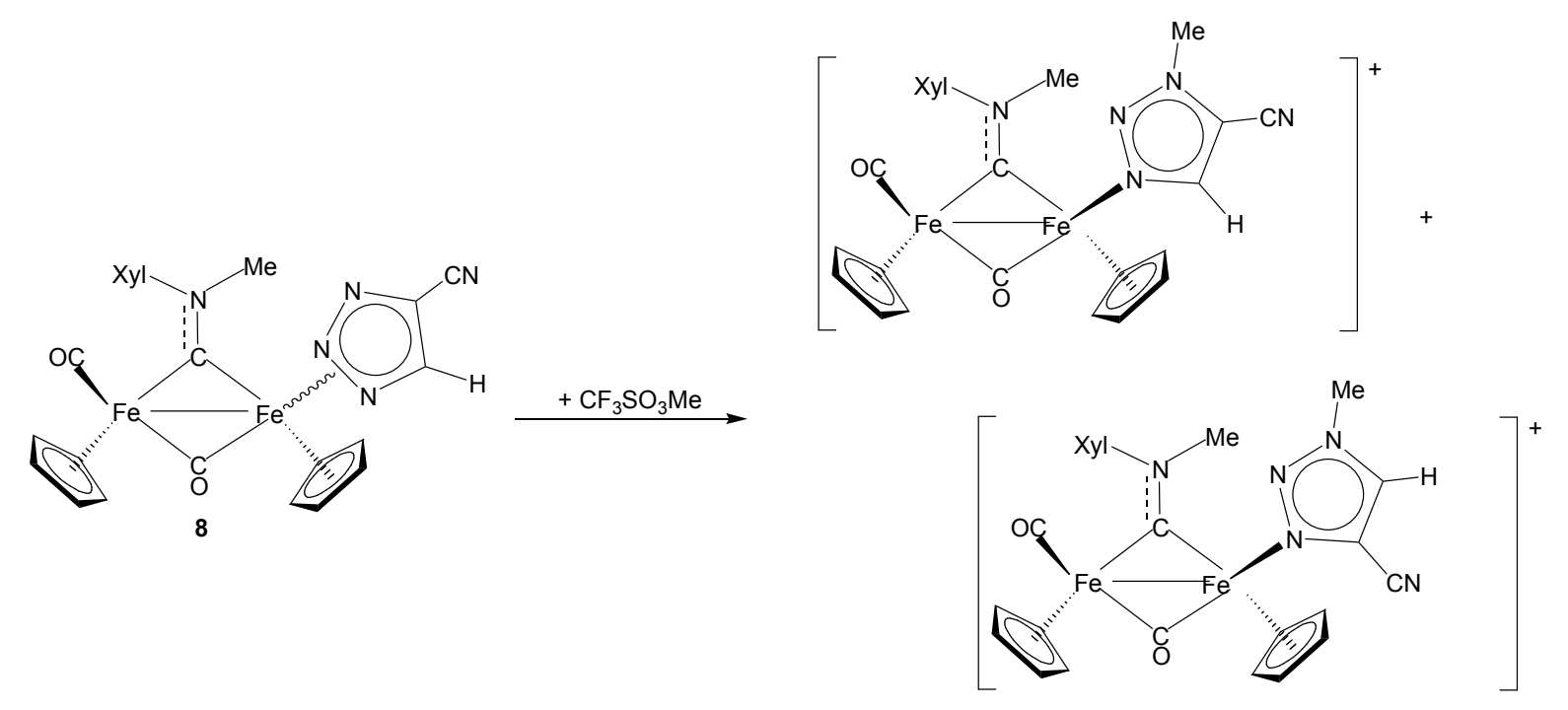

\section{Conclusions}

This study shows that dimetallic aminocarbyne complexes containing pseudohalide ligands can be obtained by $\mathrm{MeCN}$ displacement from $\left[\mathrm{M}_{2}\{\mu-\mathrm{CN}(\mathrm{Me})(\mathrm{R})\}(\mu-\right.$ $\left.\mathrm{CO})(\mathrm{CO})(\mathrm{MeCN})(\mathrm{Cp})_{2}\right]\left[\mathrm{SO}_{3} \mathrm{CF}_{3}\right](\mathrm{M}=\mathrm{Fe}, \mathrm{R}=\mathrm{Xyl}, \mathbf{1 a} ; \mathrm{M}=\mathrm{Fe}, \mathrm{Me}, \mathbf{1 b} ; \mathrm{M}=\mathrm{Ru}, \mathrm{R}=\mathrm{Xyl}, \mathbf{1}$; $\mathrm{M}=$ $\mathrm{Ru}, \mathrm{R}=\mathrm{Me}, \mathbf{1 d}$ ). We had previously shown that nitrile substitution in $\mathbf{1}$ could be used also for the introduction of other N-donor ligands, such as amines and imines [18]. All these reactions are completely selective, and no product of addition of the N-nucleophile has been observed during our work, even though nucleophilic additions to coordinated nitriles have been widely documented in the literature [23]. Probably, the MeCN ligand in $\mathbf{1}$ is not sufficiently activated to react with Nnucleophiles, whereas it reacts easily with C-nucleophiles [9a, 9c, 10c].

The results reported in this paper suggest that nitrile substitution in $\mathbf{1}$ proceeds with inversion of the configuration of the metal-centre and, in fact, the products obtained at room temperature present a trans geometry of the Cp ligands, whereas the parent complexes $\mathbf{1}$ have a cis structure. Moreover, trans to cis isomerisation occurs at high temperature, indicating that the trans isomer is the kinetic product whereas the cis species is the thermodynamic one, as previously found for the vinyliminium complexes $\left[\mathrm{Fe}_{2}\left\{\mu-\sigma: \eta^{3}-\mathrm{C}\left(\mathrm{R}^{\prime}\right)=\mathrm{C}\left(\mathrm{R}^{\prime}\right) \mathrm{C}=\mathrm{N}(\mathrm{Me})(\mathrm{R})\right\}(\mu-\right.$ $\left.\mathrm{CO})(\mathrm{CO})(\mathrm{Cp})_{2}\right]\left[\mathrm{SO}_{3} \mathrm{CF}_{3}\right][10 \mathrm{a}, 10 \mathrm{~b}]$. 


\section{Supplementary material}

Crystallographic data for the structural analyses have been deposited with the Cambridge Crystallographic Data Centre, CCDC Nos. 253688 for trans-2b, 253689 for $\mathbf{4 b} \cdot \mathbf{C H}_{\mathbf{2}} \mathbf{C l} \mathbf{2}, 253690$ for 5a, 253691 for $\mathbf{6 b} \cdot \mathbf{0 . 5 C} \mathbf{C H}_{2} \mathbf{C l}_{\mathbf{2}}$ and 253692 for $\mathbf{8} \cdot \mathbf{C H}_{\mathbf{2}} \mathbf{C l}_{2}$. Copies of this information can be obtained free of charge from the Director, CCDC, 12 Union Road, Cambridge CB2 1EZ, UK (fax: +441233-336033; e-mail: deposit@ccdc.cam.ac.uk or http://www.ccdc.cam.ac.uk).

\section{Acknowledgements.}

We thank the Ministero dell’Università e della Ricerca Scientifica e Tecnologica (MIUR) (project: "New strategies for the control of reactions: interactions of molecular fragments with metallic sites in unconventional species") and the University of Bologna ("Funds for Selected Research Topics") for financial support.

\section{References}

[1] (a) P. M. Secondo, J. M. Land, R. G. Baughman, H. L. Collier, Inorg. Chim. Acta. 309 (2000) 13; (b) K. B. Shiu, W. N. Guo, T. J. Chan, J. C. Wang, L. S. Liou, S. M. Peng, M. C. Cheng, Organometallics 14 (1995) 1732; (c) R. Vicente, A. Escuer, J. Ribas, X. Solans, Inorg. Chem. 3 (1992) 1726; (d) R. Cortes, J. L. Pizzarro, L. Lezama, M. I. Arriortua, T. Rojo, Inorg. Chem. 33 (1994) 2697.

[2] J. L. Burmeister, Coord. Chem. Rev. 105 (1990) 77.

[3] (a) A. H. Norbury, Advances in Inorganic Chemistry and Radiochemistry, Academic Press, New York, 1975, p. 17; (b) R. A. Bailey, S. L. Kozak, T. W. Michelsen, W. N. Mills, Coord. Chem. Rev. 6 (1971) 407; (c) A. M. Golub, H. Kohler, V. V. Skopenko, Chemistry of Pseudo-halides, Elsevier, New York, 1986.

[4] (a) J. Nelson, S. M. Nelson, J. Chem. Soc. (1969) 1597; (b) G. Thiele, P. Hilfrich, Z. Naturforsch., Teil B 30 (1975) 19.

[5] S. J. Anderson, D. S. Brown, K. J. Finney, J. Chem. Soc., Dalton Trans. (1979) 152.

[6] (a) H. W. Fruhauf, Chem. Rev. 97 (1997) 523; (b) Z. Dori, R. F . Ziolo, Chem. Rev. 73 (1973) 247.

[7] C. W. Chang, G. H. Lee, Organometallics 22 (2003) 3107. 
[8] (a) V. G. Albano, L. Busetto, C. Camiletti, C. Castellari, M. Monari, V. Zanotti, J. Chem. Soc., Dalton Trans. (1997) 4671; (b) V. G. Albano, S. Bordoni, L. Busetto, C. Camiletti, M. Monari, A. Palazzi, F. Prestopino, V. Zanotti, J. Chem. Soc., Dalton Trans. (1997) 4665.

[9] (a) V. G Albano,. L. Busetto, F. Marchetti, M. Monari, V. Zanotti, J. Organomet Chem. 649 (2002) 64; (b) V. G. Albano, L. Busetto, M. Monari, V. Zanotti, J. Organomet. Chem. 606 (2000) 163; (c) V. G. Albano, S. Bordoni, L. Busetto, F. Marchetti, M. Monari, V. Zanotti, J.Organomet. Chem. 684 (2003) 37.

[10] (a) V. G. Albano, L. Busetto, F. Marchetti, M. Monari, S. Zacchini, V. Zanotti, Organometallics 22 (2003) 1326; (b) V. G. Albano, L. Busetto, F. Marchetti, M. Monari, S. Zacchini, V. Zanotti, J. Organomet. Chem. 689 (2004) 528; (c) L. Busetto, F. Marchett, S. Zacchini, V. Zanotti, Eur. J. Inorg. Chem. (2004) 1494.

[11] W. Wilker, D. Leibfritz, R. Kerssebaum, W. Beimel, Magn. Reson. Chem. 31 (1993) 287.

[12] K. Stott, J. Stonehouse, J. Keeler, T. L. Hwang, A. J. Shaka, J. Am. Chem. Soc. 117 (1995) 4199.

[13] SMART \& SAINT Software Reference Manuals, Version 5.051, Bruker Analytical X-Ray Instruments Inc., Madison, Wi, 1998.

[14] G. M. Sheldrick, SADABS, Program for empirical absorption correction, University of Göttingen, Germany, 1996.

[15] G. M. Sheldrick, SHELX97, Program for crystal structure determination, University of Göttingen, Germany, 1997.

[16] J. E. Huheey, Inorganic Chemistry, Third Edition, Harper International SI edition, 1983, p. 513.

[17] T. E. Sloan, A. Wojcicki, Inorg. Chem. 7 (1968) 1268.

[18] L. Busetto, F. Marchetti, S. Zacchini, V. Zanotti, E: Zoli, J. Organomet. Chem. Accepted for Publication

[19] M. A. Bush, G. A. Sim, J. Chem. Soc (A) (1970) 605.

[20] (a) P. Paul, K. Nag, Inorg. Chem. 26 (1987) 2969; (b) W. Rigby, P. M. Bailey, J. A. McCleverty, P. M. Maitlis, J. Chem. Soc., Dalton Trans. (1979) 371; (c) T. Kemmerich, J. H. Nelson, N. E. Takach, H. Boeheme, B. Jablonski, W. Beck, Inorg. Chem. 21 (1982) 1226.

[21] (a) J. H. Nelson, D. L. Schmitt, R. A. Henty, D. W. Moore, H. B. Jonassen, Inorg. Chem. 9 (1970) 2678; (b) D. A. Redfield, J. H. Nelson, R. A. Henry, D. W. Moore, H. B. Jonassen, J. Am. Chem. Soc. 96 (1974) 6298; (c) R. L. Kieft, W. M. Peterson, G. L. Blundell, S. Horton, R. A. Henry, H. B. Jonassen, Inorg. Chem. 15 (1976) 1721.

[22] P. H. Kreutzer, J. C. Weis, H. Bock, J. Erbe, W. Beck, Chem. Ber. 116 (1983) 2691. 
[23] (a) R. A. Michelin, M. Mozzon, R. Bertani, Coord. Chem. Rev. 147 (1996) 299; (b) V. Y. Kukushkin, A. J. L. Pombeiro, Chem. Rev. 102 (2002) 1771. 


\section{Graphical abstract}

Diiron and diruthenium aminocarbyne complexes containing pseudohalide ligands (i.e. $\mathrm{NCS}^{-}, \mathrm{NCO}^{-}, \mathrm{N}_{3}^{-}$) have been obtained by nitrile replacement; both cis and trans isomers can be formed depending on the reaction temperature and trans to cis isomerisation is observed at high temperature. The terminal azido ligand undergoes 1,3-dipolar cycloaddition reactions with alkynes and fumaronitrile, affording new dinuclear triazolato complexes.

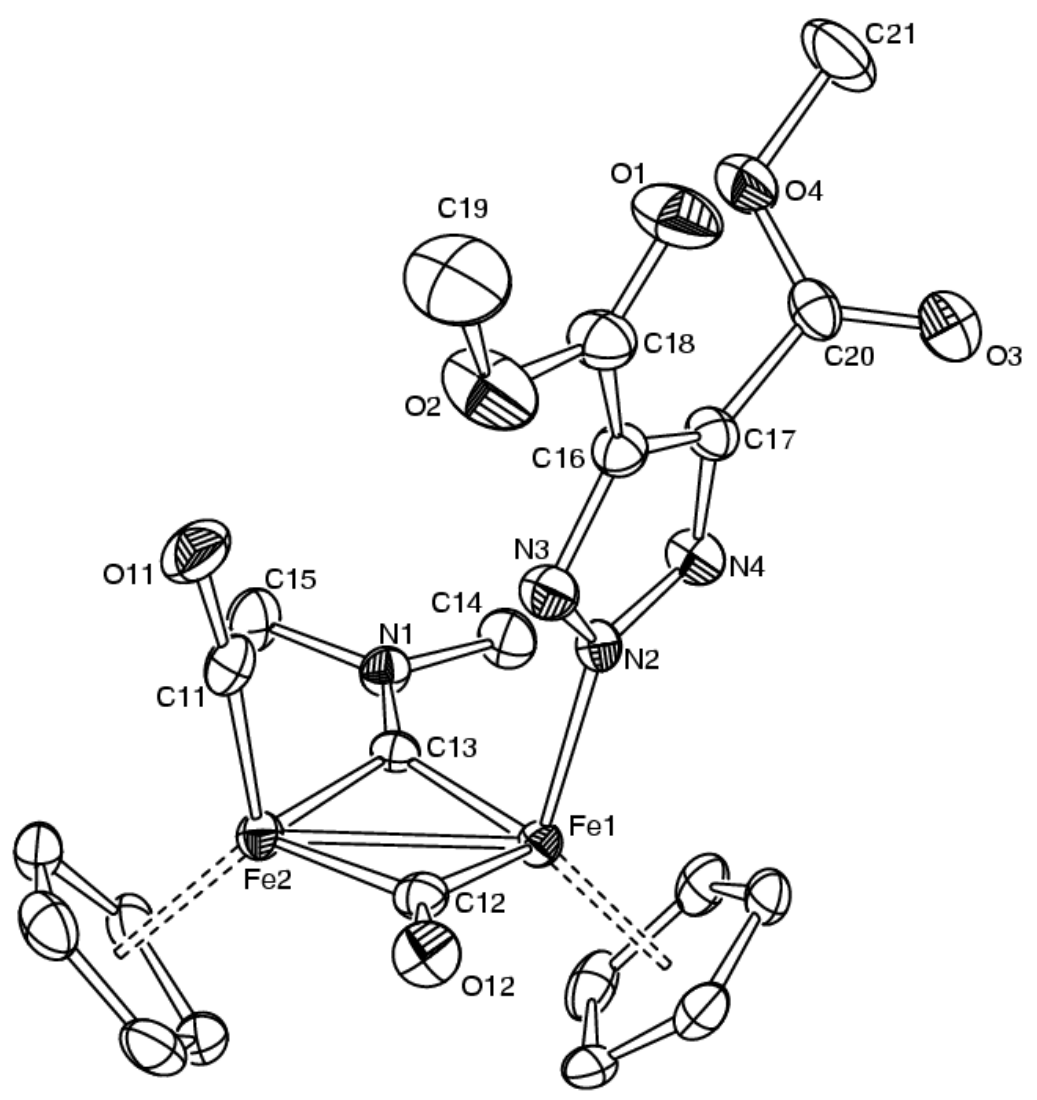

